\title{
Lagrangian spheres, symplectic surfaces and the symplectic mapping class group
}

\author{
TIAN-JUN Li \\ WEIWEI WU
}

\begin{abstract}
Given a Lagrangian sphere in a symplectic 4-manifold $(M, \omega)$ with $b^{+}=1$, we find embedded symplectic surfaces intersecting it minimally. When the Kodaira dimension $\kappa$ of $(M, \omega)$ is $-\infty$, this minimal intersection property turns out to be very powerful for both the uniqueness and existence problems of Lagrangian spheres. On the uniqueness side, for a symplectic rational manifold and any class which is not characteristic, we show that homologous Lagrangian spheres are smoothly isotopic, and when the Euler number is less than 8, we generalize Hind and Evans' Hamiltonian uniqueness in the monotone case. On the existence side, when $\kappa=-\infty$, we give a characterization of classes represented by Lagrangian spheres, which enables us to describe the non-Torelli part of the symplectic mapping class group.
\end{abstract}

53D05, 53D12, 53D42

\section{Introduction}

For a symplectic 4-manifold $(M, \omega)$, symplectic surfaces and Lagrangian surfaces are of complementary dimensions. Thus we can ask what can be said about their intersection pattern. Welschinger investigated this problem for a Lagrangian torus $L$ in [54], where he proves that the class $[L]$ pairs trivially with any effective class, and a symplectic sphere with positive Chern number can be isotoped symplectically away from $L$.

In the case when $L$ is a Lagrangian sphere in $S^{2} \times S^{2}$ with a product symplectic form, Hind [22] constructed two transverse foliations of symplectic spheres where each sphere intersects $L$ in a single point. This is used to show that every such $L$ is Hamiltonian isotopic to the antidiagonal. For a Lagrangian sphere $L$ in a symplectic Del Pezzo surface with Euler number at most 7, Evans showed in [13] that it can be displaced from certain symplectic spheres with positive Chern number up to Hamiltonian isotopy, and applied this displacement result to prove the uniqueness of Hamiltonian isotopy class of Lagrangian spheres. 
In Section 3, we generalize Evans' displacement result in two ways, the first being:

Theorem 1.1 Let $L$ be a Lagrangian sphere in a symplectic 4-manifold $(M, \omega)$, and $A \in H_{2}(M ; \mathbb{Z})$ with $A^{2} \geq-1$. Suppose $A$ is represented by a symplectic sphere $C$. Then $C$ can be isotoped symplectically to another representative of $A$ which intersects $L$ minimally.

In this paper all surfaces are smooth, embedded, connected and oriented. We say that two closed surfaces intersect minimally if they intersect transversely at $|k|$ points, where $k$ is the homological intersection number.

The second generalization is for symplectic surfaces of arbitrary genus in manifolds with $b^{+}=1$. To state it let $\mathcal{E}_{\omega}$ be the set of $\omega$-exceptional classes

$$
\mathcal{E}_{\omega}=\left\{E \in H_{2}(M, \mathbb{Z}): E \text { is represented by an } \omega \text {-symplectic }(-1) \text { sphere }\right\} .
$$

Theorem 1.2 Suppose $(M, \omega)$ is a symplectic 4-manifold with $b^{+}=1$ and $L$ is a Lagrangian sphere. Assume $A \in H_{2}(M, \mathbb{Z})$ satisfies $\omega(A)>0, A^{2}>0$ and $A \cdot E \geq 0$ for all $E \in \mathcal{E}_{\omega}$. Then there exists a symplectic surface in the class $n A$ intersecting $L$ minimally for large $n \in \mathbb{N}$.

These theorems on minimal intersection are this paper's main innovation. Theorem 1.2 is proved by combining the symplectic Seiberg-Witten theory with the symplectic field theory. The symplectic Seiberg-Witten theory produces embedded, connected pseudo-holomorphic submanifolds for a class of compatible almost complex structures suitable for applying symplectic field theory. Via neck stretching, the symplectic field theory then produces in the limit the desired symplectic surfaces which intersect $L$ minimally. When applying the symplectic field theory, one important step is to establish Lemma 3.8, which also plays a crucial role in the proof of Theorem 1.1.

One consequence of Theorem 1.2 is that we are able to effectively perform the Lagrangian-relative inflation procedure when $b^{+}=1$ (Section 5).

This turns out to be useful in dealing with a variety of questions, especially the existence of Lagrangian spheres. To approach this question, it is convenient to introduce the following definition.

Definition 1.3 A class $\xi$ is called $K_{\omega}$-null spherical if $\xi^{2}=-2, K_{\omega}(\xi)=0$ and it is represented by a smooth sphere. Here $K_{\omega}$ is the symplectic canonical class. 
We classify $K$-null spherical classes in any $(M, \omega)$ with $\kappa=-\infty$. Recall that $\kappa(M, \omega)$ is the Kodaira dimension of $(M, \omega)$ (see for example Li [33]). The Kodaira dimension $\kappa$ takes values in the set $\{-\infty, 0,1,2\}$, and $\kappa(M, \omega)=-\infty$ exactly when $(M, \omega)$ is symplectic rational or ruled. The classification of $K_{\omega}$-null spherical classes, together with the Lagrangian-relative inflation, enables us to further show that the obvious necessary condition for the existence of a Lagrangian sphere in $(M, \omega)$ is also sufficient.

Theorem 1.4 Let $(M, \omega)$ be a symplectic $4-$ manifold with $\kappa=-\infty$. The class $\xi \in \mathrm{H}_{2}(M ; \mathbb{Z})$ is represented by a Lagrangian sphere if and only if $\xi$ is $K_{\omega}$-null spherical and $\omega(\xi)=0$.

On the other hand, as in [13], Theorem 1.1 is useful in establishing uniqueness results for rational manifolds. A rational manifold is $\mathbb{C} P^{2} \# k \overline{\mathbb{C P}}^{2}$ or $S^{2} \times S^{2}$. When $M$ is a rational manifold, $(M, \omega)$ is called a symplectic rational manifold. A symplectic rational manifold $(M, \omega)$ which is monotone, ie $[\omega]=K_{\omega}$, is also called a symplectic Del Pezzo surface.

Theorem 1.5 Let $(M, \omega)$ be a symplectic rational manifold with Euler number $\chi \leq 7$, and $\xi$ a $K_{\omega}$-null spherical class with $\omega(\xi)=0$. If $\xi$ is not characteristic when $\chi=6$, then Lagrangian spheres in $\xi$ are unique up to Hamiltonian isotopy.

Hind [22] proved this in the case of $S^{2} \times S^{2}$ and Evans [13] for symplectic Del Pezzo surfaces with Euler number up to 7 . Notice that this is equivalent to the transitivity of the Hamiltonian group action on the space of homologous Lagrangian spheres. The proof of Theorem 1.5 will be presented in Section 6. Recall that a class $\xi$ is called characteristic if $\xi \cdot u=u \cdot u$ modulo 2 for any $u \in H_{2}(M, \mathbb{Z})$. We believe that uniqueness still holds when $\chi=6$ and $\xi$ is characteristic. However, the condition $\chi \leq 7$ in Theorem 1.5 is necessary, as demonstrated by Seidel's twisted Lagrangian spheres in symplectic Del Pezzo surfaces with $\chi \geq 8$ [47].

Further, we prove:

Theorem 1.6 Let $(M, \omega)$ be a symplectic rational manifold and $\xi$ a $K_{\omega}$-null spherical class with $\omega(\xi)=0$. If $\xi$ is not characteristic when $\chi=6$, then Lagrangian spheres in $\xi$ are unique up to smooth isotopy.

In the monotone case this was again due to Evans [14]. We expect the extra condition of being noncharacteristic when $\chi=6$ will eventually be removed. In fact, we are not aware of examples of homologous but not smoothly isotopic Lagrangian spheres in any 
symplectic 4-manifolds. For Lagrangian tori, such examples in a primitive homology class were first constructed by Vidussi in [53], and null-homologous ones were further constructed by Fintushel and Stern in [16].

We also conjecture the following version of uniqueness.

Conjecture 1.7 For any two homologous Lagrangian spheres $L_{1}$ and $L_{2}$ in a symplectic rational manifold $(M, \omega)$, there exists $\phi \in \operatorname{Symp}_{h}(M, \omega)$ such that $\phi\left(L_{1}\right)=L_{2}$.

In other words, the Torelli part $\operatorname{Symp}_{h}(M, \omega)$, which is the subgroup of $\operatorname{Symp}(M, \omega)$ acting trivially on homology, should also act transitively on the space of Lagrangian spheres in a fixed homology class. Evans [15] calculated explicitly the homotopy type of $\operatorname{Symp}_{h}(M, \omega)$ when $(M, \omega)$ is a symplectic Del Pezzo surface with $\chi \leq 8$ (also known to Pinnsonault). In particular, when $\chi \leq 7$, it is connected thus agreeing with $\operatorname{Ham}(M, \omega)$. In our upcoming work [38] we will extend the connectedness to the nonmonotone case.

It turns out that we are able to calculate the non-Torelli part of the symplectic mapping class group from Theorem 1.4. Recall that each Lagrangian sphere $L$ gives rise to a symplectomorphism, well defined up to isotopy (see [47] and Section 2.1.1), which is denoted by $\tau_{L}$ and called the Lagrangian Dehn twist along $L$.

Theorem 1.8 Let $(M, \omega)$ be a symplectic 4 -manifold with $\kappa=-\infty$. Then the homological action of $\operatorname{Symp}(M, \omega)$ is generated by Lagrangian Dehn twists. In other words, for any $f \in \operatorname{Symp}(M, \omega)$, there are Lagrangian spheres $L_{i}$ such that $f_{*}=$ $\left(\tau_{L_{1}}\right)_{*} \circ\left(\tau_{L_{2}}\right)_{*} \circ \cdots \circ\left(\tau_{L_{r}}\right)_{*}$.

At the homological level, Theorem 1.8 could be viewed as a symplectic version of a classical theorem of $\mathrm{M}$ Noether, which asserts that a birational automorphism of $\mathbb{C} P^{2}$ (also known as a plane Cremona map) can be decomposed into a series of ordinary quadratic transformations (see Alberich-Carramiñana [2] for a complete account).

After the paper was completed, we received a manuscript by V V Shevchishin [49], where he also proved Theorems 1.4 and 1.8 (see [49, Theorem $4^{\prime}$ (ii)]) using a completely different approach via genus 0 Lefschetz fibrations. Further, the Coxeter system for the group $\Gamma_{W}\left(\omega^{*}\right)$, which encodes the homological action of the symplectomorphism group, is explicitly presented in Theorem $4^{\prime}$ (ii).

Acknowledgements The authors would like to thank Richard Hind for his interest in our work and innumerable inspiring comments, as well as pointing out an error in an earlier draft. We would also like to thank Robert Gompf, Jonathan Evans, Chris Wendl, Ke Zhu, Weiyi Zhang and Chung-I Ho for helpful conversations. We also thank Liz Storm for an exceptional editorial job. The authors are supported by FRG 0244663. 


\section{SFT of Lagrangian $S^{2}$}

\subsection{Geometry of $T^{*} S^{2}$}

We first recall some standard facts of $T^{*} S^{2}$. Consider the embedding of the unit sphere in $\mathbb{R}^{3}$, which induces a symplectic embedding of $T^{*} S^{2}$ into $T^{*} \mathbb{R}^{3}=\mathbb{R}^{3} \times \mathbb{R}^{3}$. In terms of the coordinates $(u, v) \in \mathbb{R}^{3} \times \mathbb{R}^{3}, T^{*} S^{2}$ is thus given by equations [47; 13]

$$
\left.\left\{(u, v) \in \mathbb{R}^{3} \times \mathbb{R}^{3}:|u|=1, u \cdot v=0\right)\right\},
$$

and the symplectic form is the restriction of $\omega_{\text {can }}=d \lambda_{\text {can }}=\sum d v_{j} d u_{j}$ on $\mathbb{R}^{6}$, where the Liouville form $\lambda_{\text {can }}=\sum v_{j} d u_{j}$ is also well-defined. (2-1) provides a Lagrangian splitting of the tangent bundle of $T^{*} S^{2}$ into the horizontal $u$-direction and the vertical $v$-direction.

Here is another useful model. Consider the affine quadric $Q=\left\{z_{1}^{2}+z_{2}^{2}+z_{3}^{2}=1\right\} \subset \mathbb{C}^{3}$. In terms of $u=\operatorname{Re} z \in \mathbb{R}^{3}$ and $v=\operatorname{Im} z \in \mathbb{R}^{3}, Q$ is described by $|u|^{2}-|v|^{2}=1, u \cdot v=0$. Therefore $(u, v) \rightarrow(-u /|u|, v|u|)$ is a diffeomorphism from $Q$ to $T^{*} S^{2}$. Moreover, if we restrict $\omega_{\text {can }}$ on $\mathbb{R}^{6}$ to $Q$, the diffeomorphism is in fact a symplectomorphism.

2.1.1 Symplectomorphisms of $T^{*} S^{2}$ The symplectomorphism group of $T^{*} S^{2}$ contains some compact subgroups. For each $l>0$, denote by $T_{l}^{*} S^{2}$ the open disk bundle with $|v|<l$, and $H_{l}$ the sphere bundle of length $l$. The isometry group $\mathrm{SO}$ (3) of $S^{2}$ acts on $\left(T^{*} S^{2}, \omega_{\text {can }}\right)$ as symplectomorphisms preserving each $H_{l}$.

The Hamiltonian function $Z(u, v)=\frac{1}{2}|v|^{2}$ generates a circle action on $T^{*} S^{2}$, agreeing with the cogeodesic flow. If we apply the symplectic cut operation in Lerman [30] to $\overline{T_{l}^{*} S^{2}}$ along $H_{l}$, we obtain $S^{2} \times S^{2}$ with a monotone symplectic form (see for example Audin [3]). In other words, $T_{1}^{*} S^{2}$ embeds into a monotone $S^{2} \times S^{2}$ as the complement of the diagonal $\Delta$.

The mapping class group of the compactly supported symplectomorphism group of $\left(T^{*} S^{2}, \omega_{\text {can }}\right)$ is nontrivial. In fact, it is the infinite cyclic group generated by a model Dehn twist of the zero section; see Seidel [46].

To define the model Dehn twist, consider the Hamiltonian function $T(u, v)=|v|$ on $T^{*} S^{2} \backslash$ zero section\}, whose Hamiltonian vector field is the unit field $(v /|v|, 0)$. The induced circle action is

$$
\sigma_{t}(u, v)=(\cos (t) u+\sin (t)(v /|v|), \cos (t) v-\sin (t)|v| u) .
$$

Notice that $\sigma_{\pi}$ is the antipodal map $A(u, v)=(-u,-v)$, which extends smoothly over the zero section. Now choose a function $\rho: \mathbb{R} \rightarrow \mathbb{R}$ satisfying $\rho(t)=0$ for $t \gg 0$ and 
$\rho(-t)=\rho(t)-t$. The Hamiltonian flow of $\rho(T)$ is $\sigma_{t \rho^{\prime}(|v|)}(u, v)$. Since $\rho^{\prime}(0)=1 / 2$, the time $2 \pi$ map extends smoothly over the zero section as the antipodal map. The resulting compactly supported symplectomorphism $\tau(u, v)$ of $T^{*} S^{2}$ is called a model Dehn twist.

There is a smooth isotopy with compact support from $\tau^{2}$ to the identity, but no such symplectic isotopies exist.

2.1.2 Contact geometry of sphere bundles The length $l$ sphere bundle $H_{l}=$ $\{|v|=l\}$ is a contact manifold with contact form $\lambda_{\text {can }}$. At the point $(u, v)$ the contact plane distribution $\xi=\operatorname{ker} \lambda_{\text {can }}$ is spanned by $(u \times v, 0)$ and $(0, u \times v)$.

The Reeb vector field at $(u, v)$ is the vector field $(v, 0)$. Thus there are two dimensional simple Reeb orbits, all with the same period, and they foliate $H_{l}$. This is a special case of a Reeb flow of Morse-Bott type. In particular, the Reeb flow agrees with the cogeodesic flow of $S^{2}$ with round metric.

The vector fields $(u \times v, 0)$ and $(0, u \times v)$ provide a global trivialization $\Phi$ of $\xi$. With respect to $\Phi$, the action of the Reeb flow on $\xi$ along any Reeb orbit in $H_{l}$ is considered as a path of matrices in $\operatorname{sp}(2, \mathbb{R})$, whose Maslov index is defined to be the Conley-Zehnder index of the orbit [12] (see also Salamon and Zehnder [45]). From the calculation in [22] (see also [13]), simple Reeb orbits have Conley-Zehnder index 2.

The manifold $H_{l}$ is in fact a contact-type hypersurface in $T_{l+\epsilon}^{*} S^{2}$, where the Liouville vector field is $(0, v)$. In particular, $\overline{T_{l}^{*} S^{2}}=\{|v| \leq l\}$ is a Liouville domain with convex boundary $H_{l}$.

2.1.3 Cylindrical coordinates To apply SFT, we need to change to cylindrical coordinates. Consider a diffeomorphism $\Psi: T^{*} S^{2} \rightarrow T^{*} S^{2},(u, v) \rightarrow(u, \psi(|v|) v /|v|)$, where $\psi:[0, \infty) \rightarrow[0, \infty)$ is a smooth increasing function such that $\psi(s)=s$ for $s$ small, and $\psi(s)=e^{s}$ for $s>r . \Psi$ is the identity near the zero section, and $\left(T^{*} S^{2}, \Psi^{*} \omega_{\text {can }}\right)$ is a symplectic manifold with one positive cylindrical end. Let $\omega=\Psi^{*} \omega_{\text {can }}$.

Then $\left(T_{l}^{*} S^{2}, \omega\right)$ is still a Liouville domain, with the Liouville field given by the unit field $\eta=(0, v /|v|)$ for $|v|>r$. Moreover, $\left(T^{*} S^{2}, \omega\right)$ is the (cylindrical) symplectic completion of $\left(T_{l}^{*} S^{2}, \omega\right)$.

On $H_{l}$, the contact form is $\lambda_{l}=(\psi(l) / l) \lambda$, and the Reeb vector field at $(u, v)$ is $R_{l}=(l / \psi(l) v, 0)$. 


\subsection{Lagrangian $S^{2}$ and good almost complex structures}

Let $L \subset(M, \omega)$ be a Lagrangian two sphere. From the Weinstein neighborhood theorem, the Lagrangian sphere $L$ has a neighborhood $U$ symplectomorphic to $\left(T_{2 r}^{*} S^{2}, \omega_{\text {can }}\right)$ for some small $r>0$. Denote the symplectomorphism by $\Xi$. Let $U_{l}=\Xi^{-1}\left(\overline{T_{l}^{*} S^{2}}\right)$ for $l<2 r$, and $W_{l}=M \backslash U_{l}$ be the complement of $U_{l}$.

In particular, $H=\partial U_{l}$ is a contact-type hypersurface with contact form $\lambda=\Xi_{*}^{-1} \lambda_{l}$.

2.2.1 $J_{t}^{0}$ on $T^{*} S^{2}$ Following [22], we make a specific choice of $\omega$-compatible almost complex structure $J^{0}$ on $T^{*} S^{2}$ as follows: near the zero section, $J^{0}(X, 0)=$ $(0, X)$; and for $|v|>r$,

$$
\left.J^{0}\right|_{(u, v)}(v, 0)=(0,(\psi(l) / l) v),\left.\quad J^{0}\right|_{(u, v)}(u \times v, 0)=(0, u \times v) .
$$

The structure $J^{0}$ is $\mathrm{SO}(3)$-invariant, and $J^{0}$ is adjusted in the sense that, for $|v|>r$, it is $\frac{\partial}{\partial s}$-invariant, sending the Liouville field to the Reeb field.

Choose $l \in(r, 2 r)$. When restricted to the Liouville domain $\left(\overline{T_{l}^{*} S^{2}}, \omega\right), J^{0} \mid \overline{T_{l}^{*} S^{2}}$ is adjusted in the collar neighborhood $r<|v| \leq l$, and its cylindrical completion is canonically identified with $\left(T^{*} S^{2}, J^{0}\right)$.

We need to further consider a deformation $J_{t}^{0}$ of $J^{0}$. Let $V_{t}=[-t-\epsilon, t+\epsilon]$ and $\beta_{t}: V_{t} \rightarrow[-\epsilon, \epsilon]$ be a strictly increasing function with $\beta_{t}(s)=s+t$ on $[-t-\epsilon,-t-\epsilon / 2]$ and $\beta_{t}(s)=s-t$ on $[t+\epsilon / 2, t+\epsilon]$. Define a smooth embedding $f_{t}: V_{t} \times H_{l} \rightarrow T^{*} S^{2}$ by

$$
f_{t}(s, m)=\left(\beta_{t}(s)+l, m\right) .
$$

Let $\bar{J}_{\underline{t}}$ be the $\frac{\partial}{\partial s}$-invariant almost complex structure on $V_{t} \times H_{l}$ such that $\bar{J}_{t}\left(\frac{\partial}{\partial s}\right)=R_{l}$ and $\left.\bar{J}_{t}\right|_{\xi}=\left.J^{0}\right|_{\xi}$. Glue the almost complex manifold $\left(T^{*} S^{2} \backslash f_{t}\left(V_{t} \times H_{l}\right), J^{0}\right)$ to $\left(V_{t} \times H_{l}, \bar{J}_{t}\right)$ via $f_{t}$ to obtain the family of almost complex structures $J_{t}^{0}$ on $T^{*} S^{2}$.

Notice that each $J_{t}^{0}$ agrees with $J^{0}$ away from the collar $l-\epsilon<|v|<l+\epsilon$. And on this collar, it agrees with $J^{0}$ on $\xi$, while

$$
\left.J_{t}^{0}\right|_{(u, v)}(v, 0)=\left(0,\left.\frac{d \beta_{t}^{-1}}{d s}\right|_{s=|v|-l} \frac{\psi(l)}{l} v\right) .
$$

On the other hand, via $f_{t}, J_{t}^{0}$ restricted to $\overline{T_{l}^{*} S^{2}}$ is the same as $J^{0}$ on $\overline{T_{l+t}^{*} S^{2}}$. In particular, $J_{\infty}^{0}$ can be viewed an almost complex structure on $T^{*} S^{2}$, which is in fact equal to $J^{0}$. 
2.2.2 Neck-stretching on $M$ We say that an almost complex structure $J$ on $M$ is adjusted to $H=\partial U_{l}$ with respect to the Liouville vector field $\Xi_{*}^{-1}(\eta)$, if in a tubular neighborhood of $H, J$ is invariant under the flow $\Xi_{*}^{-1}(\eta), J\left(\Xi_{*}^{-1}(\eta)\right)$ is the Reeb vector field on $H$, and $J$ preserves the contact plane field $\zeta$ defined by the contact structure $i_{\eta} \omega$.

Following [14] consider the following Fréchet manifold of adjusted almost complex structures:

$$
\overline{\mathcal{J}}=\left\{J \in \mathcal{J}_{\omega}: J=\Xi_{*}^{-1} J^{0} \text { on } U\right\}
$$

Given $J \in \overline{\mathcal{J}}$, define

$$
J_{t}=J \text { on } X \backslash U, \quad J_{t}=\Xi_{*}^{-1} J_{t}^{0} \text { on } U .
$$

Notice that $J_{t}$ is in fact the neck-stretching of the adjusted $J$ along $\partial U_{l}$ with respect to $\Xi_{*}^{-1}(\eta)$. Fix a sequence $\left\{t_{i} \in \mathbb{R}: t_{i} \rightarrow+\infty\right\}$, we further define a sequence of Fréchet manifolds of adjusted almost complex structures:

$$
\overline{\mathcal{J}}(i)=\left\{J \in \mathcal{J}_{\omega}: J=\Xi_{*}^{-1} J_{t_{i}}^{0} \text { in } U\right\} .
$$

From the explicit description of $J_{t_{i}}$ in 2.2.1, we can reverse the neck-stretching, thus there is a diffeomorphism $P_{i}: \overline{\mathcal{J}}(i) \rightarrow \overline{\mathcal{J}}$.

When $i \rightarrow \infty$ the neck-stretching process results in an almost complex structure $J_{\infty}$ on the union of symplectic completions $\bar{W}$ and $\bar{U}$ of $W$ and $U_{r} . \bar{W}$ and $\bar{U}$ are two open symplectic manifolds with cylindrical ends, with $\left(\bar{U}, J^{\infty}\right)$ being $\left(T^{*} S^{2}, J^{0}\right)$. The structure $J_{\infty}$ on the cylindrical end of $\bar{W}$ can be described explicitly: one simply extends $\eta$ in the obvious way, and endows an $\eta$-adjusted almost complex structure which still restricts to $J$ on $\zeta$ as above.

To describe the limits of pseudo-holomorphic curves under the deformation $J_{t}$, we need another open symplectic manifold. Let $S H$ be the symplectization of the contact manifold $H$. We endow $S H$ again the $\eta$-adjusted almost complex structure as on the cylindrical ends of $\bar{W}$ and $\bar{U}$, and also denote it by $J_{\infty}$.

\subsection{Finite energy holomorphic curves}

Suppose $S$ is a closed Riemann surface and $\Gamma \subset S$ an ordered finite set of punctures. Let $(Z, \omega)$ be any of the three symplectic 4-manifolds $\bar{W}, \bar{U}$, or $S H$, each equipped with the adjusted almost complex structure $J_{\infty}$. Denote $E^{+}\left(E^{-}\right)$to be the positive (negative) end, which is allowed to be empty. 
Notice that, since $J_{\infty}^{0}\left(\frac{\partial}{\partial s}\right)=R_{l}$, and $\zeta$ is $J_{\infty}^{0}$-invariant, the real trivialization $\Phi$ of $\zeta$ on $H_{l}$ canonically induces a complex trivialization of the complex rank 2 bundle $\left(T Z, J_{\infty}\right)$ along $E^{ \pm}$, which we still denote by $\Phi$.

Suppose $u: S \backslash \Gamma \rightarrow Z$ is a proper map. We call $u$ simple if it does not factor through a multiple cover.

Let $u^{ \pm}$be the restriction to $u^{-1}\left(E^{ \pm}\right)$. Then $u^{ \pm}$has the form $\left(a_{ \pm}, v_{ \pm}\right)$in coordinates $\mathbb{R}_{ \pm} \times H$. Consider the set $\mathcal{C}$ of functions $\phi_{ \pm}: \mathbb{R}_{ \pm} \rightarrow \mathbb{R}$ with integral 1 .

The $\lambda$-energy of a map $u$ : $S \backslash \Gamma \rightarrow Z$ is defined by

$$
E_{\lambda}(u)=\sup _{\phi_{ \pm} \in \mathcal{C}}\left(\int_{u^{-1}\left(E^{+}\right)}\left(\phi_{+} \circ a_{+}\right) d a_{+} \wedge v_{+}^{*} \lambda+\int_{u^{-1}\left(E^{-}\right)}\left(\phi_{-} \circ a_{-}\right) d a_{-} \wedge v_{-}^{*} \lambda\right) .
$$

The energy of $u$ is then given by

$$
E(u)=\int_{u^{-1}\left(Z \backslash\left(E^{+} \cup E^{-}\right)\right)} u^{*} \omega+E_{\lambda}(u)
$$

and $u$ is called a finite energy map if $E(u)<\infty$. Since we are in the Morse-Bott situation, ie the Reeb flow on $E^{ \pm}$is Morse-Bott, finite energy $J_{\infty}$-holomorphic curves are asymptotic to periodic orbits in $E^{ \pm}$; see Bourgeois [9].

Suppose $S$ has genus $g$, and $u$ has $s^{+}$positive punctures converging to $\gamma_{k}^{+}, 1 \leq k \leq s^{+}$, $s^{-}$negative punctures converging to $\gamma_{k}^{-}, 1 \leq k \leq s^{-}$. Two such maps $u$ and $u^{\prime}$ are called equivalent if there is a biholomorphism $h:(S, \Gamma) \rightarrow\left(S^{\prime}, \Gamma^{\prime}\right)$ such that $u=u^{\prime} \circ h$.

Each $u$ is associated with a CR operator, and $u$ is called (SFT) regular if the operator is surjective [13]. Denote the index of this operator by index $(u)$. To state the index formula, suppose $n_{i}^{+}=\operatorname{cov}\left(\gamma_{i}^{+}\right)$and $n_{j}^{-}=\operatorname{cov}\left(\gamma_{j}^{-}\right)$, where $\operatorname{cov}(\gamma)$ denotes the multiplicity of $\gamma$ over a simple Reeb orbit. Since each Reeb orbit is in a 2 dimensional manifold and has CZ index 2, following the computation on [22; 9], we have

(2-3) index $(u)=-(2-2 g)+2\left(s^{+}+s^{-}\right)+2 c_{1}^{\Phi}([u])+\sum_{k=1}^{s^{+}} 2 \operatorname{cov}\left(\gamma_{k}^{+}\right)-\sum_{k=1}^{s^{-}} 2 \operatorname{cov}\left(\gamma_{k}^{-}\right)$.

Here $c_{1}^{\Phi}(T Z)$ is the relative first Chern class of $\left(T Z, J_{\infty}\right)$ relative to the trivialization $\Phi$ along the ends, $[u]$ is the relative homology class of $u$ [13].

The following is a very special case of a theorem due to Wendl, which states that for certain $u$, the SFT regularity is automatic. 
Theorem 2.1 (Wendl [55]) Suppose $(W, J)$ is a 4-dimensional almost complex manifold with cylindrical end modelled on contact manifolds foliated by Morse-Bott Reeb orbits, and $u:(S, \Gamma) \rightarrow W$ is a embedded pseudo-holomorphic curve with punctures. If

$$
\operatorname{index}(u)>2 g+2|\Gamma|-2,
$$

then $u$ is regular.

\subsubsection{Regular holomorphic curves in $\bar{W}$ We discuss the SFT transversality in $\bar{W}$.}

Remark 2.2 It is well-known, for example by [42, Remark 3.2.3] that, to achieve transversality for the moduli space of pseudo-holomorphic curves, it suffices to consider the space of $\omega$-compatible almost complex structure which is fixed on an open set, provided that every pseudo-holomorphic curve representing the class passes through its complement.

Recall that a Baire set is the countable intersection of open and dense sets. Since no punctured pseudo-holomorphic curves can lie completely inside $\bar{U}$, the arguments to prove [13, Theorem 5.22] also proves:

Proposition 2.3 Using notation in Section 2.2, there exists a Baire set in $\overline{\mathcal{J}}_{W} \subset \overline{\mathcal{J}}$ such that for any $J \in \overline{\mathcal{J}}_{W}, J_{\infty}$ is SFT regular in the sense that every finite energy simple $J_{\infty}$-holomorphic curve $u$ is regular.

We will need variations of other standard transversality results about pseudo-holomorphic curves, where the above observation will be crucial.

2.3.2 Genus 0 curves in $S H$ with a single simple asymptote In $S H$ we will encounter curves as in the following lemma.

Lemma 2.4 Suppose $u: C \rightarrow S H$ is a $J_{\infty}$-holomorphic curve of genus 0 in $S H$ with one positive end asymptotic to a simple Reeb orbit. Then $u$ is a trivial cylinder.

Proof The proof is contained in [13, Lemma 7.5] (see also [22; 10]). We briefly recall the main points. Since each Reeb orbit is nontrivial in $\pi_{1}(H)$ and $C$ has genus 0 , there has to be at least one negative puncture. On the other hand, since $E_{\lambda}(u) \geq 0$ and all Reeb orbits have the same period, $u$ has at most one negative puncture, which has to be simple. Thus $u$ is a trivial cylinder. 
2.3.3 $J^{0}$-Holomorphic planes in $T^{*} S^{2}$ In $T^{*} S^{2}$ we need to consider embedded holomorphic planes with one (positive) end asymptotic to a simple Reeb orbit.

As mentioned, on $T^{*} S^{2}, J_{\infty}$ is the same as $J^{0}$. Notice that $J^{0}$ interchanges the two summands of the Lagrangian splitting of the tangent bundle of $T^{*} S^{2}$. Thus $\operatorname{det}\left(T T^{*} S^{2}, J^{0}\right)$ is canonically trivialized since the Lagrangian horizontal two plane bundle is orientable. The expected dimension of the moduli space of embedded $J^{0}$ holomorphic plane $u$ with one (positive) end asymptotic to a simple Reeb orbit is thus given by

$$
\operatorname{index}(u)=-2+2+2=2 .
$$

This follows from the general index formula (2-3), and the vanishing of $c_{1}^{\Phi}$ for all punctured curves in $T^{*} S^{2}$.

It is proved in [22, Lemmas 8-9, Section 4] that if $\widetilde{J}^{0}$ is close to $J^{0}$ and any embedded $\widetilde{J}^{0}$-holomorphic planes with one simple puncture is regular, then $\widetilde{J}^{0}$ enjoys the following properties:

(1) There are two $\widetilde{J}^{0}$-foliations $\mathcal{F}_{\alpha}$ and $\mathcal{F}_{\beta}$ in $T^{*} S^{2}$, such that there is a one-one correspondence from simple Reeb orbits to planes in each foliation.

(2) Each element in $\mathcal{F}_{\alpha}\left(\mathcal{F}_{\beta}\right.$, resp.) intersects the zero-section at a single point positively (negatively, resp.).

We will call the planes in $\mathcal{F}_{\alpha}\left(\mathcal{F}_{\beta}\right.$, resp.) $\alpha$-planes ( $\beta$-planes, resp.).

One consequence of (2-5) is that we can appeal to Wendl's Theorem 2.1 to conclude that each embedded $J^{0}$-holomorphic planes with one simple puncture is regular. In particular, $J^{0}$ also satisfies the above properties. Furthermore, we have:

Lemma 2.5 A $J^{0}$-holomorphic plane in $T^{*} S^{2}$ asymptotic to a simple Reeb orbit belongs to either $\mathcal{F}_{\alpha}$ or $\mathcal{F}_{\beta}$. Moreover, an $\alpha$-plane and a $\beta$-plane intersect transversally if they do not share the same asymptote.

Proof The proof is largely similar to [22, Lemma 8]. One could think of $T^{*} S^{2}$ topologically as a neighborhood of $\bar{\Delta}$, the antidiagonal in $S^{2} \times S^{2}$. The complement is then a disk bundle over $\Delta$ the diagonal, of which the boundary of disk fibers coincides with the simple Reeb orbits in $T^{*} S^{2}$. One can then glue these disks to elements in $\mathcal{F}_{\alpha}$ and $\mathcal{F}_{\beta}$, resulting in two foliations in $S^{2} \times S^{2}$, with classes $\left[S^{2} \times \mathrm{pt}\right]$ and $\left[\mathrm{pt} \times S^{2}\right]$, respectively. Suppose we have a $J^{0}$-holomorphic plane $P$ in $U$ asymptotic to some simple Reeb orbit $\gamma$, which does not belong to either $\mathcal{F}_{\alpha}$ nor $\mathcal{F}_{\beta}$, it must intersect some $P_{\alpha} \in \mathcal{F}_{\alpha}$ and $P_{\beta} \in \mathcal{F}_{\beta}$ positively, where $P_{\alpha}$ and $P_{\beta}$ have asymptotes $\gamma_{\alpha}, \gamma_{\beta}$ 
which are different from $\gamma$. Now $P, P_{\alpha}$ and $P_{\beta}$ can all be capped in $S^{2} \times S^{2}$ by the above procedure, resulting in three spheres intersecting only in $U$. By construction, the sphere formed by capping $P$ has positively intersection with both $\left[S^{2} \times \mathrm{pt}\right]$ and $\left[\mathrm{pt} \times S^{2}\right]$, but intersects $\Delta$ at a single point, which leads to a contradiction.

The second assertion can be proved similarly, for if $\gamma_{\alpha} \neq \gamma_{\beta}$, the capped sphere does not have intersection in the complement of $T^{*} S^{2}$, so they must intersect inside $T^{*} S^{2}$ for homological reason.

Remark 2.6 If we do not appeal to Wendl's automatic transversality result, instead of $J^{0}$, we could simply use a fixed $\widetilde{J}^{0}$ satisfying the properties above throughout the paper.

2.3.4 SFT compactness Following [22] we briefly recall the relevant compactness results in the symplectic field theory adapted to our case. For detailed expositions on the subject, we refer the readers to Bourgeois, Eliashberg, Hofer, Wysocki and Zehnder [10] and Bourgeois [9].

Let $M_{\infty}=\bar{W} \cup S H \cup \bar{U}$, and $J_{\infty}$ be the almost complex structure defined as in Section 2.2. Let $\Sigma$ be a Riemann surface with nodes. A level- $k$ holomorphic building consists of the following data:

(i) (Level) A labelling of the components of $\Sigma \backslash$ nodes $\}$ by integers $\{1, \ldots, k\}$ which are the levels. Two components sharing a node differ at most by 1 in levels. Let $\Sigma_{r}$ be the union of the components of $\Sigma \backslash$ nnodes\} with label $r$.

(ii) (Asymptotic matching) Finite energy holomorphic curves $v_{1}: \Sigma_{1} \rightarrow U$ and $v_{r}: \Sigma_{r} \rightarrow S H, 2 \leq r \leq k-1, v_{k}: \Sigma_{k} \rightarrow W$. Any node shared by $\Sigma_{l}$ and $\Sigma_{l+1}$ for $1 \leq l \leq k-1$ is a positive puncture for $v_{l}$ and a negative puncture for $v_{l+1}$ asymptotic to the same Reeb orbit $\gamma . v_{l}$ should also extend continuously across each node within $\Sigma_{l}$.

Now for a given stretching family $\left\{J_{t_{i}}\right\}$ as previously described, as well as $J_{t_{i}}$-curves $u_{i}: S \rightarrow\left(M, J_{t_{i}}\right)$, we define the Gromov-Hofer convergence as follows:

A sequence of $J_{t_{i}}$-curves $u_{i}: S \rightarrow\left(M, J_{t_{i}}\right)$ is said to be convergent to a level-k holomorphic building $v$ in Gromov and Hofer's sense, using the above notation, if there is a sequence of maps $\phi_{i}: S \rightarrow \Sigma$, and for each $i$, there is a sequence of $k-2$ real numbers $t_{i}^{r}, r=2, \ldots, k-1$, such that

(i) (Domain) $\phi_{i}$ are locally biholomorphic except that they may collapse circles in $S$ to nodes of $\Sigma$,

(ii) (Map) the sequences $u_{i} \circ \phi_{i}^{-1}: \Sigma_{1} \rightarrow U, u_{i} \circ \phi_{i}^{-1}+t_{i}^{r}: \Sigma_{r} \rightarrow S H, 2 \leq r \leq k-1$, and $u_{i} \circ \phi_{i}^{-1}: \Sigma_{k} \rightarrow W$ converge in $C^{\infty}$-topology to corresponding maps $v_{r}$ on compact sets of $\Sigma_{r}$. 
Now the celebrated compactness result in SFT reads:

Theorem 2.7 [10] If $u_{i}$ has a fixed homology class, there is a subsequence $t_{i_{m}}$ of $t_{i}$ such that $u_{t_{i m}}$ converges to a level- $k$ holomorphic building in the Gromov and Hofer's sense.

\section{Minimal intersection}

In this section we prove Theorem 1.1 and Theorem 1.2. There are two main ingredients, the symplectic Seiberg-Witten theory which produces embedded, connected pseudoholomorphic submanifolds for a class of compatible almost complex structures suitable for applying symplectic field theory. Via neck stretching the symplectic field theory then produces in the limit the desired symplectic surfaces which intersect $L$ minimally.

\subsection{Embedded and nodal pseudo-holomorphic submanifolds}

We first introduce some notation. All surfaces in this section are closed. Given a class $e \in H_{2}(M, \mathbb{Z})$, let $\eta_{\omega}(e)$ be the $\omega$-symplectic genus of $e$ :

$$
\eta_{\omega}(e)=\frac{e \cdot e+K_{\omega}(e)+2}{2} .
$$

This is exactly the genus of a connected embedded $\omega$-symplectic surface in class $e$ (if there is one) from the adjunction formula.

Also define the dimension of $e$ as

$$
d(e)=\frac{-K_{\omega}(e)+e \cdot e}{2} .
$$

The quantity $d(e)$ is the expected dimension of the moduli space of embedded pseudoholomorphic curve of genus $\eta_{\omega}(e)$ in the class $e$. In terms of $\eta_{\omega}(e), d(e)$ can also be expressed as

$$
d(e)=-K_{\omega}(e)+\eta_{\omega}(e)-1 .
$$

Suppose $C$ is a compact, connected, pseudo-holomorphic submanifold of $M$. Then $C$ has the structure of a Riemann surface and it represents a nonzero class $[C]$. Moreover, there is a canonically associated elliptic operator

$$
D_{C}: \Gamma(N) \rightarrow \Gamma\left(N \otimes T^{1,0} C\right),
$$

where $N$ is the normal bundle of $C$. This operator $D_{C}$ is called the normal operator of $C$ and the index of $D_{C}$ is exactly given by $d([C])$. 
Fix a set $\Omega$ of $d([C])$ distinct points. If $\Omega \subset C$, then we can define the operator

$$
D_{C} \oplus \mathrm{ev}_{\Omega}: \Gamma(N) \rightarrow \Gamma\left(N \otimes T^{1,0} C\right) \oplus\left(\left.\oplus_{p \in \Omega} N\right|_{p}\right) .
$$

The index of $D_{C} \oplus \mathrm{ev}_{\Omega}$ is 0 . And the kernel of $D_{C} \oplus \mathrm{ev}_{\Omega}$ should be thought of as giving a sort of Zariski tangent space to the space of pseudo-holomorphic embeddings of $C$ in $M$ containing the subset $\Omega$ (as a point in the space of smooth embeddings). We call $C(J, \Omega)$ nondegenerate if the operator $D_{C} \oplus \mathrm{ev}_{\Omega}$ has trivial cokernel (and also trivial kernel).

$D_{C}$ is a real CR operator on $(C, N)$. For such operators, there is the following automatic transversality result.

Theorem 3.1 (Hofer-Lizan-Sikorav [25]; Ivashkovich-Shevchishin [26]) Let $\Sigma$ be a Riemann surface of genus $g$, and let $L$ be a complex line bundle over $\Sigma$. Let $D$ be a real CR operator. Suppose $c_{1}(L) \geq 2 g-1$, then coker $D=0$.

We will show in the next two subsections that in two situations, given a class $e$, there is a Baire set of pairs $(J, \Omega)$ for which there are connected $J$-holomorphic submanifolds of genus $\eta_{\omega}(e)$ through $\Omega$. The Baire property is shown by first setting up universal models of various type of pseudo-holomorphic curves, and then exploiting the Fredholm properties of $D$ in conjunction with the Sard-Smale theorem and the Gromov compactness theorem to rule out unwanted behavior for generic pairs $(J, \Omega)$.

We also need to generalize to the case of a nodal pseudo-holomorphic submanifold in the sense of Sikorav [50]. Let $\Sigma=\cup \Sigma_{i}$ be a nodal Riemann surface, where $\Sigma_{i}$ are the irreducible components. A $J$-holomorphic map $f: \Sigma \rightarrow(M, J)$ is said to be nodal if $f$ has distinct tangents along two branches at each node. For our purpose, we call a nodal curve $f$ a nodal submanifold if $f$ is an embedding on each $\Sigma_{i}$. Thus a nodal submanifold is a union of embedded submanifolds intersecting transversally. Let $C_{i}=f\left(\Sigma_{i}\right)$.

For a nodal submanifold, the analogue of (3-3), $D \cup C_{i}$, is defined in [50, Section 4] in terms of the normalization of $\Sigma . D \cup C_{i}$ is elliptic and its index is simply given by $\sum_{i} d\left(\left[C_{i}\right]\right)$.

In this case, for each $i$, fix a subset $\Omega_{i} \subset C_{i}$ with $d\left(\left[C_{i}\right]\right)$ distinct points and not containing any of the nodes. Then the operator $D \cup C_{i} \oplus \operatorname{ev} \cup \Omega_{i}$ is an elliptic operator with index zero, and $f$ is called nondegenerate if $D \cup C_{i} \oplus \operatorname{ev} \cup \Omega_{i}$ has trivial cokernel. The automatic transversality in this context, [50, Corollary 2] implies $D \cup C_{i} \oplus \operatorname{ev} \cup \Omega_{i}$ is onto if

$-K_{\omega}\left(\left[C_{i}\right]\right)>0$ for each $i$. 
3.1.1 Symplectic spheres Suppose $C$ is an embedded symplectic sphere with selfintersection at least -1 . In this case

$$
d([C])=-K_{\omega}([C])-1, \quad[C] \cdot[C]=-K_{\omega}([C])-2 .
$$

The following should be well known. We present some details in view of the generalization to certain configurations, Proposition 3.4.

Proposition 3.2 Let $(M, \omega)$ be a symplectic 4-manifold and $e \in H_{2}(M ; \mathbb{Z})$ with $e^{2} \geq-1$ a class represented by an embedded symplectic sphere $C$. Then there is a path connected Baire subset $\mathcal{T}_{e}$ of $\mathcal{J}_{\omega} \times M_{d(e)}$ such that a pair $(J, \Omega)$ lies in $\mathcal{T}_{e}$ if and only if there is a unique embedded $J$-holomorphic sphere in the class $e$ containing $\Omega$. Here $M_{d}$ is the space of $d$-tuples of distinct (but unlabeled) points in $M$. Consequently, any symplectic sphere in the class $e$ is isotopic to $C$.

Proof Pick an almost complex structure $J \in \mathcal{J}_{\omega}$ such that $C$ is $J$-holomorphic and $\Omega \subset C$.

Following [4, Lemma 4 and formula (15); 26], let $P=-\sum_{z_{i} \in \Omega} z_{i}$ be the divisor of $C$ and $\tilde{N}=N \otimes P$. Then there exists a real CR operator on $(C, \tilde{N})$,

$$
\widetilde{D}_{C}: \Gamma(\tilde{N}) \rightarrow \Gamma\left(\tilde{N} \otimes T^{1,0} C\right),
$$

with the property that coker $\widetilde{D}_{C} \cong \operatorname{coker}\left(D_{C} \oplus \mathrm{ev}_{\Omega}\right)$. Notice that, by (3-5),

$$
c_{1}(\tilde{N})=c_{1}(N)-d([C])=e \cdot e-d(e)=-1 .
$$

From Theorem 3.1, $\widetilde{D}$ is surjective.

Notice that $d(e) \geq 0$. Moreover, from the positivity of intersections and the fact that $e \cdot e=d(e)-1, C$ is the only connected $J$-sphere in $e$ containing $\Omega$. Since $\widetilde{D}$ is surjective, $C$ is regular with respect to $(J, \Omega)$. Thus we conclude that the genus 0 Gromov-Witten invariant of $e$ passing through $d(e)$ points is \pm 1 , in particular, nonzero.

A marked $\mathbb{P}^{1}$ is a pair $\left(\mathbb{P}^{1},\left\{z_{i}\right\}\right)$ where $\left\{z_{i}\right\}$ is a set of unordered, distinct points. Now introduce the universal genus zero moduli space $\mathcal{P}$ associated to $e$, which is the space of $J$-holomorphic embedding $u:\left(\mathbb{P}^{1},\left\{z_{i}\right\}_{i=1}^{d(e)}\right) \rightarrow(M, J)$ with $[u]=e$ for some $J \in \mathcal{J}_{\omega}$, modulo the automorphism of $\mathbb{P}^{1}$. $\mathcal{P}$ is a Frechet manifold [42]. Moreover, the natural map $\pi$ to $\mathcal{J}_{\omega} \times M_{d(e)},\left(u, J,\left\{z_{i}\right\}\right) \rightarrow\left(J,\left\{u\left(z_{i}\right)\right\}\right)$ is Fredholm. The argument above simply means that $\pi$ is an isomorphism onto its image.

Similarly, for each possible singular type $c$, introduce the auxiliary universal moduli space $\mathcal{P}_{c}$. Each $\mathcal{P}_{c}$ is again a Frechet manifold and the projection $\pi_{c}: \mathcal{P}_{c} \rightarrow \mathcal{J}_{\omega} \times M_{d(e)}$ 
is Fredholm [42] with index at most -2 . Notice that the image of $\pi$ and the union of the images of $\pi_{c}$ cover $\mathcal{J}_{\omega} \times M_{d(e)}$ by the nontriviality of the Gromov-Witten invariant. Since each $\pi_{c}$ has negative index, the complement of the image of $\pi_{c}$ is exactly the set of regular values of $\pi_{c}$, hence is Baire. This implies the image of $\pi$ is Baire.

Now we show that the image of $\pi$ is path connected. Let $\left(J^{\prime}, \Omega^{\prime}\right)$ be in the image of $\pi$. The Sard-Smale theorem implies that along a generic path $\left(J_{t}, \Omega_{t}\right)$ connecting $(J, \Omega)$ and $\left(J^{\prime}, \Omega^{\prime}\right)$, for each $t,\left(J_{t}, \Omega_{t}\right)$ is either a regular value of projections $\pi$ and $\pi_{c}$, or it is a singular value for one of the projections but the cokernel has dimension 1 . Since each $\pi_{c}$ has index -2 and $\pi$ has no singular values, each $\left(J_{t}, \Omega_{t}\right)$ lies in $\mathcal{T}$.

Finally, notice that the path connected set $\mathcal{T}$ maps onto the space of symplectic spheres in the class $e$.

For our application we need to take one step forward.

Definition 3.3 We call an ordered configuration of symplectic spheres $\cup C_{i}$ a stable spherical symplectic configuration if

(1) $\left[C_{i}\right] \cdot\left[C_{i}\right] \geq-1$ for each $i$,

(2) for any pair $i, j$ with $i \neq j,\left[C_{i}\right] \neq\left[C_{j}\right]$, and $\left[C_{i}\right] \cdot\left[C_{j}\right]=0$ or 1 ,

(3) they are simultaneously $J$-holomorphic for some $J \in \mathcal{J}$.

The homological type refers to the set of homology classes $\left[C_{i}\right]$.

Notice that, by local positivity of intersection, 2 and 3 imply that $C_{i}$ and $C_{j}$ are either disjoint or intersect transversally at one point. In particular, it is a $J$-nodal submanifold. Further, since $C_{i} \cdot C_{i} \geq-1$, the condition (3-4) is satisfied by (3-5).

If we follow the arguments above, replacing Theorem 3.1 by [50, Corollary 2], we obtain:

Proposition 3.4 Suppose there is a stable spherical symplectic configuration $\cup_{i} C_{i}$ with type $D$. Then there is a path connected Baire subset $\mathcal{T}_{D}$ of $\mathcal{J}_{\omega} \times \prod_{i} M_{d\left(\left[C_{i}\right]\right)}$ such that a pair $\left(J, \Omega_{i}\right)$ lies in $\mathcal{T}_{D}$ if and only if there is a unique embedded $J-$ holomorphic $D$-configuration with the $i$-th component containing $\Omega_{i}$. Consequently, stable spherical symplectic configurations with the same homological type are isotopic. 
3.1.2 Gromov-Taubes invariants when $b^{+}=1$ Given a class $e$ and a pair $(J, \Omega)$ in $\mathcal{J}_{\omega} \times M^{d(e)}$, introduce the set $\mathcal{H} \equiv \mathcal{H}(e, J, \Omega)$ whose elements are the unordered sets of pairs $\left\{\left(C_{k}, m_{k}\right)\right\}$ of disjoint, connected, $J$-holomorphic submanifold $C_{k} \subset M$ and positive integer $m_{k}$, which are constrained as follows:

(1) If $e_{k}$ is the fundamental class of $C_{k}$ then $d_{k} \equiv d\left(e_{k}\right) \geq 0$.

(2) If $d_{k}>0$, then $C_{k}$ contains a subset $\Omega_{k} \subset \Omega$ consisting of precisely $d_{k}$ points.

(3) The integer $m_{k}=1$ unless $C_{k}$ is a torus with trivial normal bundle.

(4) $\sum_{k} m_{k} e_{k}=e$.

Notice that (3-2) and (3-1) imply that

- the only negative square components are spheres with square -1 ,

- a square 0 component is either a sphere or a torus.

To define the Gromov-Taubes invariant of a class $e$, Taubes [51] introduced a notion of admissibility of pairs. The Gromov-Taubes invariant GT( $e)$ of $e$ is then a suitably weighted count of $\mathcal{H}(e, J, \Omega)$ for an admissible $(J, \Omega)$, which is delicate at the presence of a toroidal component with multiplicity higher than 1 . When $b^{+}=1$, we will see that there are simple homological conditions to avoid such components.

It is rather involved to fully describe the precise meaning of admissible pairs, especially at the presence of a toroidal component with multiplicity higher than 1 . In fact, in the case $d(e)=0, \Omega$ is the empty set, we are simply talking about the admissibility of $J$ alone. Furthermore, if there are no toroidal components, $J$ is admissible if $\mathcal{H}(e, J)$ is a finite set, and each submanifold in a member of $\mathcal{H}(e, J)$ is nondegenerate.

Taubes [51] also showed that the set of admissible pair is Baire. The argument is similar to the one in Proposition 3.2. In fact, by Remark 2.2, the intersection with each $\overline{\mathcal{J}}(i)$ is still Baire in $\overline{\mathcal{J}}(i)$ since $U$ contains no closed pseudo-holomorphic curve.

When $C$ is a symplectic sphere with self-intersection at least -1 , it is easy to show that $\mathrm{GT}([C])=1$ using arguments in Proposition 3.2. In general, when $b^{+}=1$, due to Taubes' SW $\Rightarrow$ GT [52] and the Seiberg-Witten wall crossing formula, there are plenty of classes with nontrivial GT invariant, and most of them are represented by connected embedded symplectic surfaces; see Li and Li [36] and also Biran [5], McDuff [40] and Li [32]:

Proposition 3.5 Let $(M, \omega)$ be a symplectic 4-manifold with $b^{+}=1$ and canonical class $K_{\omega}$. Let $A \in H_{2}(M ; \mathbb{Z})$ be a class satisfying the following properties: 
- $\quad A^{2}>0$ and $\omega(A)>0$.

- $A-\mathrm{PD}\left(K_{\omega}\right)$ is $\omega$-positive and has nonnegative square.

- $A \cdot E \geq 0$ for all $E \in \mathcal{E}_{\omega}$.

Then $A$ has nonvanishing GT invariant and $A$ is represented by a connected embedded symplectic surface.

Lemma 3.6 Let $(M, \omega)$ be a symplectic 4-manifold with $b^{+}=1$. Suppose $e \in$ $H_{2}(M ; \mathbb{Z})$ is a class with $\eta_{\omega}(e) \geq 2, e \cdot E \geq 0$ for all $E \in \mathcal{E}_{\omega}$, and $\operatorname{GT}(e) \neq 0$. Then for any admissible $(J, \Omega), A$ has a connected $J$-holomorphic representative of genus $\eta_{\omega}(e)$.

Proof Suppose $(J, \Omega)$ is admissible. Let $C$ be a $J$-holomorphic submanifold contributing to $\mathrm{GT}(e)$. The condition that $e \cdot E \geq 0$ for all $E \in \mathcal{E}_{\omega}$ ensures $C$ has no negative-square components. Since $b^{+}(M)=1$, if $C$ is disconnected, then all the components are homologous and have square 0 . Thus $C$ is either a union of spheres with square 0 , or a union of tori with square 0 . However, this contradicts the assumption that $\eta_{\omega}(e) \geq 2$ from the adjunction formula. Therefore $C$ is a connected genus $\eta_{\omega}(e)$ surface as claimed.

Furthermore, assume that $d(e) \geq 1$. Let $\left\{U_{i}\right\}_{i=1}^{d(e)}$ be a sequence of pairwise disjoint Darboux chart. We consider the class of almost complex structures $\mathcal{F}_{\left\{U_{i}\right\}} \subset \mathcal{J}_{\omega}$ which is fixed and integrable on $U_{i}$. By Remark 2.2, there is an admissible pair $\left(\widetilde{J}, \widetilde{\Omega}=\left\{x_{i}\right\}\right)$ with $\widetilde{J} \in \mathcal{F}_{\left\{U_{i}\right\}}$ and $x_{i} \in U_{i}$. In particular, there is a connected embedded $\widetilde{J}$-holomorphic curve $\widetilde{C}$ through $\left\{x_{i}\right\}$ with $[\widetilde{C}]=e$.

For any such $\widetilde{J} \in \mathcal{F}_{\left\{U_{i}\right\}}$, let $p:\left(M^{\prime}, J_{\left\{x_{i}\right\}}\right) \rightarrow(M, \widetilde{J})$ be the complex blow-up of $(M, \widetilde{J})$ at $x_{i}$. Denote each exceptional sphere by $C_{x_{i}}$ and its neighborhood corresponding to $U_{i}$ by $U_{i}^{\prime}$. One can then endow $M^{\prime}$ with a symplectic form $\omega^{\prime}$ compatible with $J_{\left\{x_{i}\right\}}$. (see [41, Lemma 7.15]). Denote also $\mathcal{F}_{\left\{U_{i}\right\}}^{\prime} \subset \mathcal{J}_{\omega^{\prime}}$ to be the corresponding set of almost complex structures.

Lemma 3.7 Given the same assumption in Lemma 3.6 and consider $\left(M^{\prime}, J_{\left\{x_{i}\right\}}\right)$ as above. Let $E_{i}=\left[C_{x_{i}}\right], i=1, \ldots, d(e)$ and $A^{\prime}=A-\sum_{1 \leq i \leq d(e)} E_{i}$. Then $\mathrm{GT}_{\omega^{\prime}}\left(A^{\prime}\right) \neq 0$, and for $J$ in a Baire subset of $\mathcal{F}_{\left\{U_{i}\right\}}, A^{\prime}$ is represented by a connected $J_{\left\{x_{i}\right\}}$-holomorphic surface of genus $\eta_{\omega}(A)$, intersecting each $C_{x_{i}}$ transversally at one point. 
Proof Now $-K_{\omega^{\prime}}\left(A^{\prime}\right)=-K_{\omega}(A)-d(e)$ and $\eta_{\omega^{\prime}}\left(A^{\prime}\right)=\eta_{\omega}(A)$. Since $d\left(A^{\prime}\right)=0$, from the blow-up formula [35, Corollary 4.4], $A^{\prime}$ also has nontrivial GT invariant.

Since the only $J^{\prime}$-holomorphic curves contained in $\cup U_{i}^{\prime}$ are $C_{x_{i}}$, by Remark 2.2, the intersection of admissible almost complex structures on $\left(M^{\prime}, \omega^{\prime}\right)$ with $\mathcal{F}_{\left\{U_{i}\right\}}^{\prime}$ is a Baire set in $\mathcal{F}_{\left\{U_{i}\right\}}^{\prime}$.

To check the generic connectedness, by Lemma 3.6 we only need to verify the homological condition $A^{\prime} \cdot E \geq 0$ for any $E \in \mathcal{E}_{\omega^{\prime}}$. But as is shown above, there is a connected embedded $\widetilde{J}$-holomorphic $\widetilde{C} \subset M$, thus its proper transformation $\tilde{C}^{\prime} \subset M^{\prime}$ is $J_{\left\{x_{i}\right\}}$ holomorphic with $\left[\widetilde{C}^{\prime}\right]=A^{\prime}$. Notice also that every $E$ has a $J_{\left\{x_{i}\right\}}$-holomorphic representative since exceptional classes always have nontrivial GW invariant. Since the genus of $\widetilde{C}^{\prime}$ is positive, it is different from any component of $E$. By positivity of intersections, we have $\left[\widetilde{C}^{\prime}\right] \cdot E \geq 0$.

Finally, since $\mathcal{F}_{\left\{U_{i}\right\}}^{\prime}$ and $\mathcal{F}_{\left\{U_{i}\right\}}$ are canonically identified via complex blowing up the $x_{i}$ and complex blowing down the $C_{x_{i}}$, we obtain the required Baire subset of $\mathcal{F}_{\left\{U_{i}\right\}}$.

\subsection{Proof of Theorems $\mathbf{1 . 1}$ and $\mathbf{1 . 2}$}

We are ready to prove Theorems 1.1 and 1.2. For the convenience of exposition, we first investigate the behavior of generic $J$-holomorphic representatives in class $A$ in neck-stretching, when the class $A$ satisfies

$$
-K_{\omega}(A)=1-\eta_{\omega}(A) .
$$

Firstly, regarding the fixed class $A$, we claim that there is a Baire set $\mathcal{J}_{\text {reg }}(A) \subset \overline{\mathcal{J}}$ such that for each $J \in \mathcal{J}_{\text {reg }}, J_{t_{i}}$ is GT admissible for each $i$, and $J_{\infty}$ is regular in the sense of SFT for $\bar{W}$. By Proposition 2.3 there is a Baire subset $\mathcal{J}_{\text {reg }}^{\prime} \subset \overline{\mathcal{J}}$, such that for $J \in \mathcal{J}_{\text {reg }}^{\prime}$, $J_{\infty}$ is SFT regular. Recall from Section 2.2.2, $\overline{\mathcal{J}}(i)=\left\{J \mid J=J_{t_{i}}^{0}\right.$ in $\left.U\right\}$ and $P_{i}$ is the identification of $\overline{\mathcal{J}}(i)$ with $\overline{\mathcal{J}}$. We have mentioned that, as all closed pseudoholomorphic curves have to pass through $M \backslash U$, there is a Baire subset $\overline{\mathcal{J}}(i)^{\prime} \subset \overline{\mathcal{J}}(i)$ such that each member is GT admissible. One then takes $\mathcal{J}_{\text {reg }}(A)=\bigcap_{n} P_{n}\left(\overline{\mathcal{J}}_{n}^{\prime}\right) \cap \mathcal{J}_{\text {reg }}^{\prime}$. Fix $J \in \mathcal{J}_{\text {reg }}(A)$. By Lemma 3.6 there is a sequence of connected embedded $J_{t_{i}}$ holomorphic submanifolds $C_{t_{i}}$. If $C_{t_{i}}$ does not intersect $L$ for some $i<\infty$, the theorem follows. Now we assume that each $C_{t_{i}}$ intersects $L$. This assumption will eventually lead to a contradiction when $[L] \cdot[C]=0$ and is automatically satisfied if $[L] \cdot[C] \neq 0$.

By Theorem 2.7, there is a $k$-leveled curve $C_{\infty}$ as a Gromov-Hofer limit of $\left\{C_{t_{i}}\right\}_{i=0}^{\infty}$ : the piece in $M \backslash U_{l}$, which we call $C_{W}$ or the $W$-part; the piece in the symplectization of $\partial U_{l}=\mathbb{R} P^{3}$ consisting of $k-2$ levels, which we call $C_{S H}$ or the $S H$-part; the piece in $U_{l}$, which we call $C_{U}$ or the $U$-part. Let us first examine the $W$-part. 
Lemma 3.8 Suppose (3-6) is satisfied. Then $C_{W}$ is a, possibly unbranched covering, irreducible genus $-\eta_{\omega}(A)$ curve, and all asymptotic Reeb orbits are simple. Moreover, let $\bar{C}_{W}$ be the underlying simple curve, then the limits of punctures of $\bar{C}_{W}$ are pairwise distinct.

Proof By the maximum principle, $C_{W}$ is nonempty. Let $u_{i}: B_{i} \rightarrow W, 1 \leq i \leq q$, be the irreducible components of $C_{W}$ and $g_{i}$ the genus of $B_{i}$. Suppose $u_{i}$ is a degree $m_{i}$ multiple cover of $\bar{u}_{i}: \bar{B}_{i} \rightarrow W$.

Notice that

$$
c_{1}^{\Phi}=0 \text { in } U \text { and } S
$$

implies that

$$
\sum_{1 \leq j \leq q} c_{1}^{\Phi}(T W)\left(\left[u_{j}\right]\right)=-K_{\omega}(A)
$$

From the description of Gromov-Hofer convergence in Section 2.3.4, we clearly have $\sum_{1 \leq j \leq q} g_{j} \leq \eta_{\omega}(A)$. (3-6) then implies that

$$
\sum_{1 \leq j \leq q} c_{1}^{\Phi}(T W)\left(\left[u_{j}\right]\right) \leq 1-\sum_{1 \leq j \leq q} g_{j} .
$$

If $q>1$, there must be some component, say $B_{1}$, with

$$
c_{1}^{\Phi}(T W)\left(\left[u_{1}\right]\right) \leq-g_{1} .
$$

By (2-3), we have

$$
\operatorname{index}\left(\bar{u}_{1}\right)=-\left(2-2 g\left(\bar{B}_{1}\right)\right)+2 \bar{s}_{1}^{-}+2 c_{1}^{\Phi}(T W)\left(\left[\bar{u}_{1}\right]\right)-\sum_{k=1}^{\bar{s}_{1}^{-}} 2 \operatorname{cov}\left(\bar{\gamma}_{k}\right) .
$$

Here $\bar{s}_{1}^{-}$is the total number of punctures of $\bar{u}_{1}$ and the $\bar{\gamma}_{k}$ are the asymptotic Reeb orbits. By our choice of $J$, index $\left(\bar{u}_{1}\right) \geq 0$, thus we must have

$$
c_{1}^{\Phi}(T W)\left(\left[\bar{u}_{1}\right]\right) \geq 1-g\left(\bar{B}_{1}\right) .
$$

Notice that $c_{1}^{\Phi}(T W)\left(\left[u_{1}\right]\right)=m_{1} c_{1}^{\Phi}(T W)\left(\left[\bar{u}_{1}\right]\right)$. Since $2 \bar{s}_{1}^{-}-\sum_{k=1}^{\bar{s}_{1}^{-}} 2 \operatorname{cov}\left(\bar{\gamma}_{k}\right) \leq 0$, by (3-8) we have

$$
g_{1} \leq m_{1}\left(g\left(\bar{B}_{1}\right)-1\right) .
$$

But this is impossible by the Riemann-Hurwitz formula

$$
\left(g_{1}-1\right) \geq m_{1}\left(g\left(\bar{B}_{1}\right)-1\right) .
$$


This contradiction shows that $C_{W}$ is irreducible, namely, given solely by $u_{1}$, when $J \in \mathcal{J}_{\text {reg }}$. By (3-10) and (3-7), we have

$$
1-\eta_{\omega}(A)=m_{1} c_{1}^{\Phi}(T W)\left(\left[\bar{u}_{1}\right]\right) \geq m_{1}\left(1-g\left(\bar{B}_{1}\right)\right) .
$$

Since $g_{1} \leq \eta_{\omega}(A)$, we have by (3-11), that

$$
\eta_{\omega}(A)=g_{1} .
$$

Notice that this also means $u_{1}$ is an unbranched covering. Now return to (3-10), we find that

$$
\operatorname{index}\left(\bar{u}_{1}\right)=2 \bar{s}_{1}^{-}-\sum_{k=1}^{\bar{s}_{1}^{-}} 2 \operatorname{cov}\left(\bar{\gamma}_{k}\right) \geq 0 .
$$

Hence we conclude that each $\bar{\gamma}_{k}$ is a simple Reeb orbit. Since $u_{1}$ is an unbranched covering, each of its puncture also converges to one of the simple Reeb orbits, $\bar{\gamma}_{k}$.

One also sees from (3-9) and (3-12) that $C_{W}$ must have genus $g$ and all asymptotes are simple.

Since the Reeb orbits form a two dimensional Morse-Bott family, the last statement follows from the transversality of puncture evaluation of $\bar{C}_{W}$ [13, Theorem 5.24].

Now we look at the $S$-part $C_{S H}$.

Lemma 3.9 Each component of $C_{S H}$ is a trivial cylinder asymptotic to a simple Reeb orbit.

Proof $C_{S H}$ has $k-2$ levels. Let $\tau_{i}: D_{i} \rightarrow S H$ be an irreducible component of first level of $C_{S H}$. Since $C_{W}$ is connected and already has genus $\eta_{\omega}(A), D_{i}$ is of genus 0 and has a unique positive puncture since the domain of $C_{\infty}$ is obtained by collapsing a genus $g$ surface. Moreover, due to the asymptotic matching condition between two levels, this unique positive puncture of $\tau_{i}$ is asymptotic to a simple Reeb orbit since all the asymptotes of $\bar{C}_{W}$ are simple. Thus $\tau_{i}$ must be a trivial cylinder by Lemma 2.4. Similarly each component in higher level of $C_{S H}$ must be a cylinder as well (In fact, there can only be one level of trivial cylinders in $C_{S H}$ by the finite automorphism requirement of $C_{\infty}$, but we do not need this more precise description).

For the $U$-part $C_{U}$, in turn, Lemma 3.9 implies that all the positive punctures of $C_{U}$ are simple due to the asymptotic matching condition between two levels. Moreover, each component $F_{i}$ is of genus 0 and has only one positive puncture, again due to the constraint $g\left(C_{\infty}\right)=g$. Thus each $F_{i}$ is a plane with one simple positive puncture. From Lemma 2.5 and Lemma 3.8, the $U$-part is a union of some $\alpha$-and $\beta$-planes. 
Lemma 3.10 If $C_{W}$ is not a multiple cover, the $U$-part consists of either all $\alpha$-planes or all $\beta$-planes.

Proof The proof is similar to [13, Lemma 7.8]. As is explained in Lemma 2.5, an $\alpha$-plane and a $\beta$-plane do not intersect only if they have the same asymptotic Reeb orbit. This must be the case to avoid self-intersection of the holomorphic building $C_{\infty}$ which contradicts the embeddedness for $C_{t_{i}}$ at some $i<\infty$.

Therefore, if the $U$-part has at least one $\alpha$-plane and one $\beta$-plane, all planes must asymptote to the same Reeb orbit. If $C_{W}$ is not a multiple cover, since the $C_{S H}$ part consists of trivial cylinders, this is impossible by the last statement of Lemma 3.8.

Proof of Theorem 1.2 It is straightforward that when $n \in \mathbb{N}$ is large, under the assumption of Theorem 1.2 the multiple class $n A$ has the following properties: $d(n A)>0$, $\mathrm{GT}(n A) \neq 0$, and it is represented by a connected symplectic surface with genus at least 2 .

We adapt Welschinger's idea in [54] and adopt the notation in Section 3.1.2 here. Choose Darboux charts $U_{i} \subset W, i=1, \ldots, d(n A)$, and consider $\mathcal{F}_{\left\{U_{i}\right\}}$ as in the paragraphs preceding Lemma 3.7. Now choose $x_{i} \in U_{i}$ and an arbitrary $J \in \mathcal{F}_{\left\{U_{i}\right\}}$, $A^{\prime}=n A-\sum_{1 \leq i \leq d(e)} E_{i}$ as in Lemma 3.7. By Lemma 3.7 and the arguments in the paragraph following (3-6), there is a Baire set $\mathcal{J}_{\text {reg }}(n A) \subset \overline{\mathcal{J}} \cap \mathcal{F}_{\left\{U_{i}\right\}}$ such that for each $J \in \mathcal{J}_{\text {reg }}(n A),\left(J_{\left\{x_{i}\right\}}\right)_{t_{j}}$ is GT admissible for each $j$ and there is a connected embedded $\left(J_{\left\{x_{i}\right\}}\right)_{t_{j}}$-holomorphic curve $C_{j}^{\prime}$ in the class $A^{\prime}$. Moreover, $\left(J_{\left\{x_{i}\right\}}\right)_{\infty}$ is regular in the sense of SFT for the symplectic completion of $p^{-1}(W)$.

Now let us analyze the limit building $C_{\infty}^{\prime}$.

Notice that $-K_{\omega}\left(A^{\prime}\right)=1-\eta_{\omega}\left(A^{\prime}\right)$, so Lemma 3.8 could be applied. Also, from the fact that $C_{x_{i}} \cap U=\varnothing$ and $A^{\prime} \cdot E_{i}=1$, we have $C_{x_{i}} \cap C_{W}^{\prime}=1$. Therefore the $W$-part of $C_{\infty}^{\prime}$ cannot be a multiple cover. Therefore, by Lemma 3.10, $C_{\infty}^{\prime}$ intersects $L$ transversally at finitely many points, where either all the local intersections are positive or all of them are negative. This implies that for some $j<\infty$, there is an embedded $\left(J_{\left\{x_{i}\right\}}\right)_{t_{j}}$-holomorphic curve $C_{t_{j}}^{\prime}$ in the class $A^{\prime}$ with the same intersection property. Notice that $C_{t_{j}}^{\prime}$ intersects transversally with each $C_{x_{i}}$ at one point. One then obtain the desired curve $C$ in the class $A$ by complex blowing down the (disjoint) exceptional curves $C_{x_{i}}$.

Remark 3.11 When $\kappa=-\infty$, given $A$ in Theorem 1.2, we can actually find a symplectic surface intersecting $L$ minimally in the class $A$, rather than $n A$ for large $n$, if we further assume that $\eta(A) \geq 2$ and $A^{2} \geq \eta(A)-1$. Here $\eta(A)$ is the symplectic 
genus (see Section 4). This is because, by [31] one could achieve the nontriviality of GT invariants as long as $A^{2} \geq \eta(A)-1$. And if the class $A$ is reduced (see Section 4 for the rational case and [31] for the general case), one only needs easily verified conditions $\eta_{\omega}(A) \geq 2$ and $A^{2} \geq \eta_{\omega}(A)-1$ since $\eta_{\omega}(A)=\eta(A)$.

Proof of Theorem 1.1 We first deal with the case of -1 sphere $C$. By Proposition 3.2, there is a Baire set $\mathcal{J}_{\text {reg }}([C]) \subset \overline{\mathcal{J}}$ such that for each $J \in \mathcal{J}_{\text {reg }}([C])$, there is a unique embedded $J_{t_{i}}$-holomorphic sphere in the class $[C]$ for each $i$, and $J_{\infty}$ is regular in the sense of SFT for $\bar{W}$. Notice that $d([C])=0$, and since $C$ has genus 0 , its $W$-part under neck-stretching does not admit a nontrivial unbranched cover. Therefore we can apply Lemma 3.10 as in the proof of Theorem 1.2 to produce a $J_{t_{i}}$-holomorphic sphere $C_{t_{i}}$ intersecting $L$ minimally. $C_{t_{i}}$ is symplectic isotopic to $C$ by the last statement of Proposition 3.2.

For a symplectic sphere $C$ with nonnegative square, we follow the strategy above by first introducing $U_{i}$ and $\mathcal{F}_{U_{i}}$. By applying Remark 2.2 and Proposition 3.2 to $M$ and $[C]$, there is a pair $\left(\tilde{J}, \widetilde{\Omega}=\left\{x_{i}\right\}\right)$ with $\widetilde{J} \in \mathcal{F}_{\left\{U_{i}\right\}}, x_{i} \in U_{i}$, and an embedded $\widetilde{J}$-holomorphic sphere $\widetilde{C}$ through $\left\{x_{i}\right\}$ with $[\widetilde{C}]=[C]$. Let $\left(M^{\prime}, J_{\left\{x_{i}\right\}}, \omega^{\prime}\right), C_{x_{i}}, E_{i}=\left[C_{x_{i}}\right], U_{i}^{\prime}$, $\mathcal{F}_{U_{i}}^{\prime}, i=1, \ldots, d(e)$ be as in Lemma 3.7. The class $A^{\prime}=[C]-\sum_{1 \leq i \leq d(e)} E_{i}$ is represented by an $\omega^{\prime}$-symplectic -1 sphere, for instance, the proper transform of $\widetilde{C}$, thus Proposition 3.2 still holds for $A^{\prime}$.

Now apply Remark 2.2 to $M^{\prime}$ and $A^{\prime}$, then Proposition 3.2 and the arguments in the first paragraph of the present subsection imply that, there is a Baire set $\mathcal{J}_{\text {reg }}([C]) \subset \overline{\mathcal{J}} \cap \mathcal{F}_{\left\{U_{i}\right\}}$ with the following property: for each $J \in \mathcal{J}_{\text {reg }}([C])$, there is a unique embedded $\left(J_{\left\{x_{i}\right\}}\right)_{t_{j}}$-holomorphic sphere $C_{t_{j}}^{\prime}$ in the class $A^{\prime}$ for each $j$, and $\left(J_{\left\{x_{i}\right\}}\right)_{\infty}$ is regular in the sense of SFT for the symplectic completion of $p^{-1}(W)$. Moreover, $C_{t_{j}}^{\prime}$ intersects transversally with each $C_{x_{i}}$ at one point.

Now, just as in the end of the proof of Theorem 1.2, for some $j, p\left(C_{t_{j}}^{\prime}\right)$ is the desired symplectic sphere in the class $A$, where $p: M^{\prime} \rightarrow M$ is the complex blowing down map. Moreover, $p\left(C_{t_{j}}^{\prime}\right)$ is symplectic isotopic to $C$ by the last statement of Proposition 3.2.

Remark 3.12 One easily sees that the above proof works for finitely many Lagrangian spheres that are pairwise disjoint. It is not clear to the authors whether the theorem holds when they do intersect.

On the other hand, by choosing subsequences successively, one may push off certain symplectic configurations. In particular, the following will be used in the proof of Theorem 1.5. 
Corollary 3.13 Let $L$ be a Lagrangian sphere in a symplectic 4-manifold $(M, \omega)$, and $D=\left\{A_{1}, \ldots, A_{n}\right\}$ a homology type of a stable spherical symplectic configuration. If each $A_{i}$ pairs trivially with $[L]$. Then there is a symplectic $D$-configuration disjoint from $L$.

Remark 3.14 Further, we expect to be able to deform a contractible family of symplectic spheres to be disjoint from a given Lagrangian sphere. Such a result would be useful in proving Conjecture 1.7 on the uniqueness up to symplectomorphism (see Remark 5.2 and Section 6.4.2). The family being contractible is necessary: as pointed out to us by R. Hind, if one takes a representative of the generator of $\pi_{1}\left(\operatorname{Symp}\left(S^{2}, \sigma\right)\right)$, the graph of this generator as a circle family of symplectic spheres in a monotone $S^{2} \times S^{2}$ cannot be isotoped away from the antidiagonal.

\section{$4 K$-Null spherical classes when $\kappa=-\infty$}

It is in general difficult to determine whether a spherical class has a Lagrangian spherical representative. We are able to completely solve this problem for rational and ruled manifolds in Section 5.2. In this section we first derive some preliminary results.

\subsection{Rational manifolds}

We fix some notation: in this section $M$ is $\mathbb{C} P^{2} \# n \overline{\mathbb{C P}}^{2}$ with $n \geq 1$. Let $\mathcal{E}$ and $\mathcal{L}$ be the sets of integral homology classes represented by smoothly embedded spheres of square -1 and -2 respectively.

An orthogonal basis $\left\{H, E_{1}, \ldots, E_{n}\right\}$ of $H_{2}(M ; \mathbb{Z})$ is called standard if $H^{2}=1$ and $E_{i} \in \mathcal{E}$. We fix a standard basis in this section.

Let $\mathcal{K}$ be the set of symplectic canonical classes of $M$. For any sequence $\left\{\delta_{i}\right\}$, $i=0, \ldots, n$ with $\delta_{i}=0$ or 1 , let $K_{\left\{\delta_{i}\right\}}$ be the Poincáre dual of

$$
-3 H+(-1)^{\delta_{1}} E_{1}+(-1)^{\delta_{2}} E_{2}-\cdots+(-1)^{\delta_{n}} E_{n} .
$$

Then $K_{\left\{\delta_{i}\right\}} \in \mathcal{K}$. When $\delta_{i}=0$ for all $i$, we simply denote it by $K_{0}$, ie

$$
K_{0}=\mathrm{PD}\left(-3 H+E_{1}+\cdots+E_{n}\right) .
$$

4.1.1 $\mathcal{E}, \mathcal{L}$, symplectic genus and $D(M)$ We review some facts about $\mathcal{E}, \mathcal{L}, D(M)$ and the notion of symplectic genus.

Let $D(M)$ be the geometric automorphism group of $M$, ie the image of the diffeomorphism group of $M$ in $\operatorname{Aut}\left(H_{2}(M ; \mathbb{Z})\right)$. We say two classes in $H_{2}(M ; \mathbb{Z})$ are equivalent if they are related by $D(M)$. 
$\mathrm{Li}$ and $\mathrm{Li}$ [31] showed that $D(M)$ is generated by a set of spherical reflections. For $\gamma \in H_{2}(M ; \mathbb{Z})$ with $\gamma^{2}=\gamma \cdot \gamma= \pm 1$ or \pm 2 , there is an automorphism $R(\gamma) \in$ $\operatorname{Aut}\left(H_{2}(M ; \mathbb{Z})\right)$ called the reflection along $\gamma$,

$$
R(\gamma)(\beta)=\beta-\frac{2(\gamma \cdot \beta)}{\gamma \cdot \gamma} \gamma
$$

If $\gamma \in \mathcal{E}$ or $\mathcal{L}$ by [17, Proposition 2.4, Chapter III], $R(\gamma) \in D(M)$, and we call it a spherical reflection.

Another fact is that $D(M)$ acts transitively on $\mathcal{K}[36]$.

To define the symplectic genus of $e \in H_{2}(M ; \mathbb{Z})$ first introduce the subset $\mathcal{K}_{e}$ of $\mathcal{K}$ :

$$
\mathcal{K}_{e}=\left\{K \in \mathcal{K} \mid \text { there is a class } \tau \in \mathcal{C}_{K} \text { such that } \tau \cdot e>0\right\} .
$$

Here $\mathcal{C}_{K}=\left\{[\omega] \mid \omega\right.$ is a symplectic form, $\left.K_{\omega}=K\right\}$ is the $K$-symplectic cone. It is shown in [36] that $\mathcal{C}_{K}$ is completely determined by the set of $K$-exceptional spherical classes

$$
\mathcal{E}_{K}=\{E \in \mathcal{E} \mid K(E)=-1\}
$$

More precisely,

$$
\mathcal{C}_{K}=\left\{\tau \in H^{2}(M ; \mathbb{R}) \mid \tau^{2}>0, \tau(E)>0 \text { for any } E \in \mathcal{E}_{K}\right\} .
$$

The following is a useful observation.

Lemma 4.1 If $\xi=a H-\sum b_{i} E_{i} \in H_{2}(M ; \mathbb{Z})$ with $a>0$ then $K_{\left\{\delta_{i}\right\}} \in \mathcal{K}_{\xi}$.

Proof Notice that for any $K_{\left\{\delta_{i}\right\}}$, one could easily find $\tau \in \mathcal{C}_{\left.K_{\{} \delta_{i}\right\}}$ by requiring $\tau(H) \gg 0$, but keeping the corresponding signs of $E_{i}$ in $\tau$ opposite to that of $K_{\left\{\delta_{i}\right\}}$. Such a construction follows from the easy observation that classes in $\mathcal{E}_{\left.K_{\{} \delta_{i}\right\}}$ are obtained by changing the corresponding signs of those in $\mathcal{E}_{K}$ and [36, Theorem 4].

By possibly even enlarging $\tau(H)$ further, since $a>0$, one could also assure that $\tau(\xi)>0$. Therefore, $K_{\left\{\delta_{i}\right\}} \in \mathcal{K}_{\xi}$.

For $K \in \mathcal{K}_{e}$ define the $K$-symplectic genus $\eta_{K}(e)$ to be $\frac{1}{2}\left(K(e)+e^{2}\right)+1$. Finally, the symplectic genus of class $e$ is defined as

$$
\eta(e)=\max _{K \in \mathcal{K}_{e}} \eta_{K}(e) .
$$

By [31, Lemma 3.2], $\eta(e)$ has the following basic properties:

(1) $\eta(e)$ is no bigger than the minimal genus of $e$, and they are both equal to $\eta_{\omega}(e)$ in (3-1) if $e$ is represented by an $\omega$-symplectic surface for some symplectic form $\omega$.

(2) Equivalent classes have the same $\eta$. 
Note that in [31] these properties are stated for classes with positive square, but the proof actually covered all cases.

We have the following assertions characterizing $\mathcal{E}$ and $\mathcal{L}$ in terms of the symplectic genus, as well as the action of $D(M)$ on $\mathcal{E}$ and $\mathcal{L}$.

Proposition 4.2 [31, Lemma 3.4, Lemma 3.6(2)] For $e$ with $e \cdot e=-1$ or -2 , $\eta(e)=0$ if and only if $e$ is not equivalent to a reduced class.

Moreover, for $e$ with $e \cdot e=-1, \eta(e)=0$ if and only if $e \in \mathcal{E}$, Any class in $\mathcal{E}$ is equivalent to either $E_{i}$ or $H-E_{i}-E_{j}$ for some $1 \leq i, j \leq n$. If $n \neq 2$, it is equivalent to $E_{i}$.

Similarly, for $e$ with $e \cdot e=-2, \eta(e)=0$ if and only if $e \in \mathcal{L}$. Any class in $\mathcal{L}$ is equivalent to either $E_{i}-E_{j}$ or $H-E_{i}-E_{j}-E_{k}$ for some $1 \leq i, j, k \leq n$. If $n \neq 3$, it is equivalent to $E_{i}-E_{j}$.

Here a class $\xi=a H-\sum_{i=1}^{n} b_{i} E_{i}$ with $a \geq 0$ and $b_{1} \geq b_{2} \geq \cdots \geq b_{n} \geq 0$ is called reduced $[18 ; 27]$ if

$$
a \geq b_{1}+b_{2}+b_{3} .
$$

4.1.2 $K$-Null spherical classes and $D_{K}(M)$ For $K \in \mathcal{K}$ let $D_{K}(M)$ be the isotropy subgroup of $K$ of the transitive action of $D(M)$ on $\mathcal{K}$. We say two classes are $K$-equivalent if they are related by $D_{K}(M)$.

By Definition $1.3, \xi \in H_{2}(M ; \mathbb{Z})$ is a $K$-null spherical class if $\xi \in \mathcal{L}$ and $K(\xi)=0$. Hence the set of $K$-null spherical classes is denoted by $\mathcal{L}_{K}$.

We now study the interactions of $\mathcal{L}_{K}$ and $D_{K}(M)$. Due to the transitivity of the action of $D(M)$ on $\mathcal{K}$ [36], we will restrict to the case $K=K_{0}$ without loss of generality.

First of all, if $\gamma \in \mathcal{L}_{K_{0}}$, then $R(\gamma) \in D_{K_{0}}(M)$, and we call it a $K_{0}$-twist.

Secondly, notice that $D_{K_{0}}$ acts on $\mathcal{E}_{K_{0}}$ and $\mathcal{L}_{K_{0}}$.

To go further, we need to understand $\mathcal{E}_{K_{0}}$. It is clear that $E_{i} \in \mathcal{E}_{K_{0}}$. Moreover, for any symplectic form $\omega$ with $K_{\omega}=K_{0}$, the GT invariant of $H$ or any $E \in \mathcal{E}_{K_{0}}$ is nontrivial. By the positivity of intersection, we have:

Lemma 4.3 Suppose $\xi=a H-\sum b_{i} E_{i}$ is in $\mathcal{E}_{K_{0}}$, then $a \geq 0$ and $b_{i} \geq 0$. If $a=0$, then $\xi=E_{i}$ for some $i$.

It is clear that reflections $R\left(E_{i}-E_{j}\right)$ and $R\left(H-E_{i}-E_{j}-E_{k}\right)$ are $K_{0}$-twists. With this understood, we see that [43, Proposition 1.2.12] can be stated as follows: 
Proposition 4.4 Any class in $\mathcal{E}_{K_{0}}$ can be transformed to either $E_{i}$ or $H-E_{i}-E_{j}$ for some $1 \leq i, j \leq n$ via $K_{0}$-twists. If $n \neq 2$, it is $K_{0}$-equivalent to $E_{i}$ via $K_{0}$-twists.

As a consequence, we have:

Corollary 4.5 Suppose $b^{-}(M)=n \geq 2$. If $\left\{E_{i}^{\prime}\right\}_{i=1}^{k}, k \leq n-2$, is an orthogonal subset of $\mathcal{E}_{K_{0}}$, then there exists $\phi \in D_{K_{0}}(M)$, generated by $K_{0}$-twists, such that $\phi\left(E_{i}^{\prime}\right)=E_{i}, 1 \leq i \leq k$.

Proof The statement is vacuous if $n \leq 2$ and easily verified for $n=3$. We apply induction on $n$. From Proposition 4.4, there exists $\widetilde{\phi} \in D_{K_{0}}(M)$ such that $\widetilde{\phi}\left(E_{1}^{\prime}\right)=E_{i}$. One then further compose the $K_{0}$-twist $f=R\left(E_{i}-E_{1}\right)$ so that $E_{1}^{\prime}$ is eventually sent to $E_{1}$. Noting that

$$
f\left(\widetilde{\phi}\left(E_{i}^{\prime}\right)\right) \cdot E_{1}=f\left(\widetilde{\phi}\left(E_{i}^{\prime}\right)\right) \cdot f\left(\widetilde{\phi}\left(E_{1}^{\prime}\right)\right)=E_{i}^{\prime} \cdot E_{1}^{\prime}=-\delta_{1 i},
$$

we are reduced to the case $n-1$ by restricting our attention to the last $(n-1)$ exceptional classes (and $k$ is reduced by 1 as well).

Remark 4.6 Note that this is not true when $k=n-1$. Take $n=2$. Then $H-E_{1}-E_{2}$ is not equivalent to $E_{1}$ or $E_{2}$ since it is characteristic but $E_{i}$ is not.

Proposition $4.7 \quad D_{K_{0}}(M)$ is generated by $K_{0}$-twists.

Proof For $\phi \in D_{K_{0}}(M)$, apply Corollary 4.5 to $\phi\left(E_{i}\right), 1 \leq i \leq n-2$, there is a $K_{0}$-twist $f$ such that $f\left(\phi\left(E_{i}\right)\right)=E_{i}$.

Consider $F_{n-1}=f\left(\phi\left(E_{n-1}\right)\right)$ and $F_{n}=f\left(\phi\left(E_{n}\right)\right) . F_{n-1}$ and $F_{n}$ are orthogonal to $E_{i}, 1 \leq i \leq n-2$, since $f\left(\phi\left(E_{j}\right)\right) \cdot E_{i}=E_{j} \cdot E_{i}=0$ for $i \leq n-2$ and $j>n-2$. It is easy to see that the only such classes in $\mathcal{E}_{K_{0}}$ are $H-E_{n-1}-E_{n}, E_{n-1}, E_{n}$. Since $F_{n-1} \cdot F_{n}=0$, it has to be that $\left\{F_{n-1}, F_{n}\right\}=\left\{E_{n-1}, E_{n}\right\}$. By composing $f$ with the $K_{0}$-twist $R\left(E_{n}-E_{n-1}\right)$ if necessary, one obtains the desired inverse of $\phi$ generated by $K_{0}$-twists, which means $\phi$ is also generated by $K_{0}$-twists.

We now prove an analogue of Proposition 4.4 for $\mathcal{L}_{K_{0}}$. We start with:

Lemma 4.8 Suppose $\xi=a H-\sum b_{i} E_{i} \in H_{2}(M ; \mathbb{Z})$ is in $\mathcal{L}_{K_{0}}$, If $a>0$ then $\eta(\xi)=\eta_{K_{0}}(\xi)$ and $b_{i} \geq 0$. 
Proof For any $\xi \in \mathcal{L}_{K_{0}}, \eta_{K_{0}}(\xi)=0$ and the minimal genus is 0 as well. By Lemma 4.1, if $\xi=a H-\sum b_{i} E_{i} \in H_{2}(M ; \mathbb{Z})$ with $a>0$, then $\eta_{K_{\left\{\delta_{i}\right\}}}(\xi)$ is defined. Recall from the minimal genus assumption and the fact that the symplectic genus is no bigger than the minimal genus, $0=\eta_{K_{0}}(\xi) \geq \eta_{\left.K_{\left\{\delta_{i}\right.}\right\}}(\xi)$ for any choice of $\left\{\delta_{i}\right\}$. But this holds only if $b_{i} \geq 0$ for all $i$, hence the conclusion follows.

Following Evans [13], we make the following definition.

Definition 4.9 A class is called binary if it is of the form $E_{i}-E_{j}$, and ternary if it is of the form $H-E_{i}-E_{j}-E_{k}, 1 \leq i, j, k \leq n$.

Clearly, binary and ternary classes are in $\mathcal{L}_{K_{0}}$. In the rest of our paper, we denote $R\left(H-E_{i}-E_{j}-E_{k}\right)$ by $\Gamma_{i j k}$ for short.

Proposition 4.10 For $\xi \in \mathcal{L}_{K_{0}}$, either $\xi$ is $K_{0}$-equivalent to a binary or ternary class. Further, if either $n \neq 3$, or $n=3$ but $\pm \xi \neq H-E_{1}-E_{2}-E_{3}$, then $\xi$ is $K_{0}$-equivalent to the binary class $E_{1}-E_{2}$.

Proof Let $\xi=a H-\sum b_{i} E_{i}$. When $a=0$ it is easy to conclude that $\xi$ is binary. Let $r$ be the number of nonzero $b_{i}$. An easy calculation verifies the case when $r \leq 3$. Thus we assume $r>3$ with $a>0$ by possibly reversing the signs of $\xi$ (simply do a reflection with respect to $\xi$ ). By Lemma 4.8 , we may assume that $b_{1} \geq b_{2} \geq \cdots \geq b_{n} \geq 0$.

Now we write down the reflection $\Gamma_{123}$ explicitly:

$$
\Gamma_{123}(\xi)=\left(2 a-b_{1}-b_{2}-b_{3}\right) H-\sum c_{i} E_{i},
$$

where $c_{i}=b_{i}$ for $i>3$.

If $2 a-b_{1}-b_{2}-b_{3}<0$, consider the class $-\Gamma_{123}(\xi) \in \mathcal{L}_{K_{0}}$. In this case, the leading coefficient of $-\Gamma_{123}(\xi)$ is bigger than 0 . However, since $r>3$, one must have $-c_{r}=-b_{r}<0$, a contradiction to Lemma 4.8. Thus, $2 a-b_{1}-b_{2}-b_{3} \geq 0$

Moreover, from Proposition 4.2, $\xi$ is not reduced hence one must have $b_{1}+b_{2}+b_{3}>a$. Combining these facts, we have

$$
0 \leq 2 a-b_{1}-b_{2}-b_{3}<a .
$$

Also notice that $\Gamma_{123}(\xi)$ verifies all conditions of Lemma 4.8, thus $c_{i}>0$ still holds. One could then repeat the above process and use induction on the coefficient $H \cdot \xi$ until $r \leq 3$ or $a=0$.

Remark 4.11 The algorithm reducing a $K$-null spherical classes is also valid for exceptional classes. In this case, one gets an explicit $K_{0}$-equivalence from an exceptional class to $E_{i}$ when $n \geq 3$ or possibly $H-E_{1}-E_{2}$ when $n=2$. This is also used in [43]. 
4.1.3 $(K, \alpha)$-Null spherical classes and $D_{K, \alpha}(M)$ In this section we fix a class $\alpha$ in the $K$-symplectic cone $\mathcal{C}_{K}$.

Definition 4.12 A $(K, \alpha)-$ null spherical class is a $K$-null spherical class which pairs trivially with $\alpha$.

Reflections $R(\xi)$, for $\xi$ a $(K, \alpha)$-null spherical class, are called $(K, \alpha)$-twists. We also define $D_{K, \alpha}(M)$ to be the subgroup of $D_{K}(M)$ preserving $\alpha$. One has the following easy observation:

Lemma 4.13 If $\phi \in D_{K}$ then

- $\phi$ induces a bijection from $\mathcal{L}_{K, \alpha}$ to $\mathcal{L}_{K, \phi^{-1}(\alpha)}$,

- $f \rightarrow \phi^{-1} \circ f \circ \phi$ defines an isomorphism from $D_{K, \alpha}$ to $D_{K, \phi^{-1}(\alpha)}$ taking $R(\xi)$ to $R(\phi(\xi))$,

- $\alpha$ has a positive lower bound on $\mathcal{E}_{K}$ which is attained by some $K$-exceptional class.

The third assertion is a consequence of Gromov compactness and the well-known fact that, for any $E \in \mathcal{E}_{K}, \mathrm{GT}(E) \neq 0$ with respect to any symplectic form $\omega$ representing $\alpha$. We are now ready to prove the following:

Proposition 4.14 $D_{\left(K_{0}, \alpha\right)}$ is generated by $\left(K_{0}, \alpha\right)-t$ wists.

Proof We will use induction on $n$ and a trick due to Pinsonnault [44]. For $n \leq 3$ this is easy to verify directly by listing all exceptional classes.

If $n \geq 3$ choose $\left\{E_{i}^{\prime}\right\}_{i=1}^{n-2} \subset \mathcal{E}_{K_{0}}$ such that $E_{1}^{\prime}$ has minimal $\alpha$-area, and $E_{i}^{\prime}$ has minimal $\alpha$-area among exceptional classes orthogonal to $E_{j}$ for all $j<i$. By Corollary 4.5, there is $\psi \in D_{K_{0}}(M)$ such that $\psi\left(E_{i}^{\prime}\right)=E_{i}$. By Lemma 4.13 we can assume that $E_{i}^{\prime}=E_{i}$, so that among the basis elements $\left\{H, E_{1}, \ldots, E_{n}\right\}, E_{1}, \ldots, E_{n-2}$ enjoys the above minimality property.

Let $f \in D_{\left(K_{0}, \alpha\right)}$. If one could find a series of $\left(K_{0}, \alpha\right)$-twists such that their composition $\phi$ satisfies $\phi \circ f\left(E_{1}\right)=E_{1}$, one can then include $\phi^{-1}$ into our decomposition of $f$. Since $E_{1}$ is orthogonal to $\phi \circ f\left(E_{i}\right)$ for $i \neq 1$, one can then use induction on these classes. Therefore it suffices to look for such a $\phi$ in the rest of the proof.

Notice first that

$$
\alpha\left(H-E_{i}-E_{j}-E_{k}\right) \geq 0, \quad i>j>k .
$$


This is clear from the construction: since the $K_{0}$-exceptional class $\left(H-E_{i}-E_{j}\right) \cdot E_{l}=$ 0 , for all $l<k$ and $k \leq n-2$, we have $\alpha\left(H-E_{i}-E_{j}\right) \geq \alpha\left(E_{k}\right)$

Assume $f\left(E_{1}\right)=a H-\sum b_{r_{i}} E_{r_{i}}$. Notice $f\left(E_{1}\right) \in \mathcal{E}_{K_{0}}$ and $\alpha\left(f\left(E_{1}\right)\right)=\alpha\left(E_{1}\right)$. If $a=0$ then $f\left(E_{1}\right)=E_{k}$ for some $k$ and $E_{1}-E_{k} \in \mathcal{L}_{K_{0}, \alpha}$. In particular, $R\left(E_{1}-E_{k}\right) \in$ $D_{K_{0}, \alpha}$ and we can choose $\phi=R\left(E_{1}-E_{k}\right)$.

If $a \neq 0$, by Lemma 4.3, $a>0$ and $b_{i} \geq 0$. Suppose $b_{r_{1}} \geq b_{r_{2}} \geq \cdots \geq b_{r_{n}} \geq 0$. Now apply $\Gamma_{r_{1}} r_{2} r_{3}$,

$$
\Gamma_{r_{1} r_{2} r_{3}}\left(f\left(E_{1}\right)\right)=f\left(E_{1}\right)+\left(a-b_{r_{1}}-b_{r_{2}}-b_{r_{3}}\right)\left(H-E_{r_{1}}-E_{r_{2}}-E_{r_{3}}\right) .
$$

From Proposition 4.2, $a-b_{r_{1}}-b_{r_{2}}-b_{r_{3}}<0$. By (4-1), $\alpha\left(H-E_{r_{1}}-E_{r_{2}}-E_{r_{3}}\right) \geq 0$, thus

$$
\alpha\left(E_{1}\right)=\alpha\left(f\left(E_{1}\right)\right) \geq \alpha\left(\Gamma_{r_{1} r_{2} r_{3}}\left(f\left(E_{1}\right)\right)\right) .
$$

By the choice of $E_{1}$, we must have $\alpha\left(H-E_{r_{1}}-E_{r_{2}}-E_{r_{3}}\right)=0$. This means that $H-E_{r_{1}}-E_{r_{2}}-E_{r_{3}} \in \mathcal{L}_{K_{0}, \alpha}$ and $\Gamma_{r_{1} r_{2} r_{3}} \in D_{K_{0}, \alpha}(M)$.

Now from Remark 4.11, by repeating the above operations we eventually have an equivalence between $E_{1}$ and $E_{k}$ for some $k$. Denote their composition by $\widetilde{\phi}$.

If $k=1$ let $\phi=\widetilde{\phi}$. If $k \neq 1$, then $\alpha\left(E_{k}\right)=\alpha\left(E_{1}\right)$ and let $\phi=R\left(E_{1}-E_{k}\right) \circ \widetilde{\phi}$.

\subsection{Irrational ruled manifolds}

It is clear that a minimal symplectic irrational ruled manifold does not admit any Lagrangian spheres. Thus, in this subsection, $M=\left(\Sigma_{h} \times S^{2}\right) \# n \overline{\mathbb{C P}}^{2}$. Any nonminimal genus $h$ ruled manifold is of this form. Define $\mathcal{E}, \mathcal{L}, \mathcal{K}, D(M)$ as above. For $K \in \mathcal{K}$ also define $D_{K}(M), \mathcal{E}_{K}, \mathcal{L}_{K}$ and $K$-null spherical class as above.

A standard homology basis consists of $\left\{T, F, E_{1}, \ldots, E_{n}\right\}$, with the following algebraic properties:

$$
T \cdot F=1, \quad T^{2}=F^{2}=T \cdot E_{i}=F \cdot E_{i}=0, \quad E_{i}^{2}=-1, \quad 1 \leq i \leq n .
$$

Geometrically, $T$ is represented by a surface with genus $h, F$ the class of a fiber, and $\left\{E_{i}\right\}$ a maximal collection of orthogonal exceptional classes in $\mathcal{E}$. The standard canonical class is then $K_{0}=\operatorname{PD}\left(-2 T+(2 h-2) F+\sum E_{i}\right)$.

The group $D(M)$ is characterized as the subgroup of $\operatorname{Aut}\left(H_{2}(M ; \mathbb{Z})\right)$ preserving $F$ up to sign [17]. Due to the transitive action of $D(M)$ on $\mathcal{K}$ shown in [36], we may again restrict to the case $K_{\omega}=K_{0}$. 


\section{Lemma 4.15 We have}

$$
\begin{aligned}
& \mathcal{E}_{K_{0}}=\left\{E_{i}, F-E_{i}, i=1, \ldots, n\right\}, \\
& \mathcal{L}_{K_{0}}=\left\{ \pm\left(F-E_{i}-E_{j}\right), \pm\left(E_{i}-E_{j}\right), 1 \leq i<j \leq n\right\} .
\end{aligned}
$$

Proof First of all, if $\xi=a T+b F+\sum c_{i} E_{i}$ is represented by a sphere, then $a=0$. This follows from the fact that a sphere does not have a nonzero degree map to a positive genus curve.

With this understood, it is easy to determine $\mathcal{E}_{K_{0}}$ and $\mathcal{L}_{K_{0}}$ using (4-2).

For $\alpha \in \mathcal{C}_{K_{0}}$ we define $D_{K_{0}, \alpha},\left(K_{0}, \alpha\right)$-twist as before.

Lemma 4.16 $D_{K_{0}, \alpha}$ is generated by $\left(K_{0}, \alpha\right)$ twists.

Proof As in the rational manifold case, we do induction on $n=b^{-}(M)+1$.

When $n=1$, since $\phi(F)= \pm F$, it is easier to see that $D_{K_{0}}$, and hence $D_{K_{0}, \alpha}$, is trivial.

In general when $n \geq 2$, for $\phi \in D_{K_{0}, \alpha}$ we consider its action on $\mathcal{E}_{K_{0}}$. Let $E$ be the exceptional class with minimal $\alpha$ area, the induction is immediate if $\phi(E) \cdot E=0$, in which case we simply compose $\phi$ with the $\left(K_{0}, \alpha\right)-$ twist $R(E-\phi(E))$ to reduce to a lower $n$ case.

Otherwise, $\phi(E)=F-E$ by Lemma 4.15. In this case $2 \alpha(E)=\omega(F)$. Since two classes $A$ and $F-A$ are either both in $\mathcal{E}_{K_{0}}$ or neither, the minimality of $\alpha(E)$ forces all other exceptional spheres to have the same area as $E$. Since $n \geq 2$, it is clear that one could send $F-E$ back to $E$ via a composition of $\left(K_{0}, \alpha\right)$-twists, for example, the $\left(K_{0}, \alpha\right)-$ twist $R\left(E^{\prime}-E\right)$ followed by $R\left(F-E^{\prime}-E\right)$, where $E^{\prime}$ is another exceptional standard basis element orthogonal to $E$. Again we are able to reduce to a lower $n$ case.

\section{Lagrangian spherical classes when $b^{+}=1$}

Theorem 1.2 allows us to effectively apply a Lagrangian-relative version of inflation procedure in this section. Together with Proposition 4.10, this enables us to classify Lagrangian spherical classes in symplectic 4 -manifolds with $\kappa=-\infty$. We also give the proof of Theorem 1.8 in Section 5.3. 


\subsection{Lagrangian relative inflation}

The inflation procedure was first introduced by Lalonde [28] and proved useful in many fundamental problems in symplectic geometry (see Lalonde and McDuff [29] for example).

The inflation construction in [28], together with Theorem 1.2, gives:

Lemma 5.1 (Inflation lemma) Let $L$ be a Lagrangian sphere in a symplectic 4manifold with $b^{+}=1$. Let $A$ be a class in $H_{2}(M ; \mathbb{Z})$ satisfying the condition in Theorem 1.2. Assume also that $A \cdot[L]=0$. Then there is a closed form $\rho$ on $M$ in class $\mathrm{PD}(A)$ supported away from $L$ so that

$$
\beta_{t}=\omega+t \rho, \quad t \geq 0,
$$

is symplectic. In particular, $L$ remains Lagrangian for any $\beta_{t}$.

The proof is straightforward: note in [28], $\rho$ is supported near a symplectic surface in class $A$. Therefore, if such a symplectic surface is disjoint from the given Lagrangian sphere $L, L$ remains Lagrangian in the course of the inflation procedure. Now Theorem 1.2 provides the desired symplectic surface.

We first apply Lemma 5.1 to study symplectic ball embeddings in the complement of a Lagrangian sphere. Biran and Cornea studied Lagrangian relative embeddings in [8] (called mixed packing there), where the size of maximal ball embeddings is found in some cases.

In our case of a Lagrangian sphere $L$ in a symplectic 4 -manifold with $b^{+}=1$, Lemma 5.1 enables us to show that packing problems in the complement of $L$ can often be answered in the same way as for the ordinary packing problems. Here is one example. Biran showed in [6] that in any closed symplectic 4-manifold with an integral symplectic form, the symplectic packing problem is stable via inflation on a Donaldson hypersurface. For a symplectic 4-manifold $(M, \omega)$ with $b^{+}=1$ and $\omega$ integral, the class $n[\omega]$ for $n$ large satisfies the conditions in Theorem 1.2 for an arbitrary given Lagrangian sphere. Thus Lemma 5.1 can be applied to such a class and it follows from the arguments in [6] that the symplectic packing problem is also stable in $(M, \omega) \backslash L$ for $M$ a closed symplectic 4-manifold with $b^{+}=1, \omega$ an integral symplectic form on $M$ and $L$ a Lagrangian sphere in $M$.

Remark 5.2 It would be useful to prove the following parameterized version of Lemma 5.1, which would be the analogue of [40, Lemma 1.1]: Given a path $\omega_{t}$, $0 \leq t \leq 1$, of symplectic forms on $M$ with $b^{+}=1$ and a sphere $L$ Lagrangian for 
each $\omega_{t}$. Let $A$ be a class in $H_{2}(M ; \mathbb{Z})$ satisfying the conditions in Theorem 1.2. Assume also that $A \cdot[L]=0$. Then there is a path $\rho_{t}$ of closed forms on $M$ in class $\operatorname{PD}(A)$ supported away from $L$ so that

$$
\beta_{t}=\omega_{t}+\kappa(t) \rho_{t}, \quad 0 \leq t \leq 1,
$$

is symplectic whenever $\kappa(t) \geq 0$. In particular, $L$ remains Lagrangian for any $\beta_{t}$.

Lemma 1.1 in [40] is actually stated for manifolds which are not of SW simple type. And it is used there to show that for this class of manifolds, the ball embedding space

$$
E_{\bar{\lambda}, k}(M, \omega)=\left\{\psi \mid \psi: \coprod_{i=1}^{k}\left(B_{4}\left(\lambda_{i}\right), \omega_{\text {std }}\right) \hookrightarrow(M, \omega)\right\}
$$

with $\bar{\lambda}=\left(\lambda_{1}, \ldots, \lambda_{k}\right)$, is connected. With observations made in [36], it is straightforward to see that Lemma 1.1 in [40] is actually valid for any manifold with $b^{+}=1$. Thus, the connectedness of $E_{\bar{\lambda}, k}(M, \omega)$ holds for any $(M, \omega)$ with $b^{+}=1$. Further, substituting it by its $L$ relative version as above in appropriate places, we would be able to obtain the connectedness of the relative ball embedding space.

\subsection{Existence of Lagrangian spheres}

In this subsection we present the proof of Theorem 1.4. We begin with some general discussions of Lagrangian spheres in a nonminimal symplectic 4 -manifold with $b^{+}=1$.

5.2.1 Nonminimal 4-manifolds with $b^{+}=1$ and $\kappa \geq 0$ We begin with the following two persistence results.

Lemma 5.3 Let $(M, \omega)$ be a symplectic 4-manifold with $b^{+}(M)=1$ and $[\omega] \in$ $H^{2}(M ; \mathbb{Q})$. Let $(\bar{M}, \bar{\omega})$ be the one point blow up of $(M, \omega)$ with size $a$, and $\iota: H_{2}(M ; \mathbb{Z}) \rightarrow H_{2}(\bar{M}, \mathbb{Z})$ the canonical injection. If $L \subset(M, \omega)$ is a Lagrangian sphere, then there is a Lagrangian sphere in $(\bar{M}, \bar{\omega})$ in the class $\iota([L])$.

Proof By the uniqueness of blow ups [40, Corollary 1.3], we can place the ball of size $a$ anywhere in $(M, \omega)$. If the ball is disjoint from $L$, we are done. Otherwise, first choose a ball of size $a^{\prime}<a$ and disjoint from $L$, we obtain a blow up $\left(\bar{M}, \bar{\omega}^{\prime}\right)$ with a Lagrangian $\bar{L}$ from $L$. Let $p: \bar{M} \rightarrow M$ be a topological blow down map which contracts the exceptional sphere. Consider the class $\beta_{l, \delta}=l\left(\left[p^{*} \omega\right]-(a+\delta) \operatorname{PD}(E)\right)$ for $\delta>0$. Clearly, $\beta_{l, \delta}([\bar{L}])=0$. Since the $K_{\bar{\omega}}$-symplectic cone $\mathcal{C}_{K_{\bar{\omega}}}$ (which is the same as $\mathcal{C}_{\boldsymbol{K}_{\bar{\omega}^{\prime}}}$ ) is open, we can assume that $\operatorname{PD}\left(\beta_{l, \delta}\right)$ is in $\mathcal{C}_{\boldsymbol{K}_{\bar{\omega}}}$ by choosing $\delta$ small. If $a+\delta$ is further assumed to be a rational number, then there exists $l \in \mathbb{Z}^{+}$such 
that $\operatorname{PD}\left(\beta_{l, \delta}\right)$ satisfies the conditions in Lemma 5.1. Applying Lemma 5.1 to such a $\operatorname{PD}\left(\beta_{l, \delta}\right)$ and $\bar{L}$, we find that $\bar{L}$ remains Lagrangian in $\left(\bar{M}, \bar{\omega}^{\prime \prime}\right)$, where $\bar{\omega}^{\prime \prime}$ is a symplectic form in the class $\left[p^{*} \omega\right]-a \mathrm{PD}(E)$ up to a rescale. The proof is finished by again invoking the uniqueness of cohomologous symplectic forms on any manifold with $b^{+}=1$ [40, Theorem 1.2; 36, Proposition 4.11].

If $E$ is the class of the exceptional sphere, this lemma can be viewed as the persistence of Lagrangian spheres under a symplectic deformation on $\bar{M}$ in the $E$ direction, which can also be proved via the inflation construction along a symplectic surface with negative self intersection as in [37].

Lemma 5.4 Let $(\bar{M}, \bar{\omega})$ be a symplectic 4-manifold with $b^{+}(\bar{M})=1,[\bar{\omega}] \in$ $H^{2}(\bar{M} ; \mathbb{Q})$. If there are two orthogonal exceptional classes $E_{1}, E_{2} \in \mathcal{E}_{\omega}$ with equal symplectic area $a$, then there is a Lagrangian sphere in the binary class $E_{1}-E_{2}$.

Proof Let us first consider a local model: the two point blow up of a standard ball with equal size $t>0$. This can be identified with the complement of a line in $\mathbb{C} P^{2} \# 2 \overline{\mathbb{C P}}^{2}$ with a symplectic form $\tau$ with $[\tau]=\operatorname{PD}\left(H-t E_{1}-t E_{2}\right)$. Notice that $\left(\mathbb{C} P^{2} \# 2 \overline{\mathbb{C P}}^{2}, \tau\right)$ is symplectomorphic to a one point blow up of a monotone $S^{2} \times S^{2}$ with size $1-2 t$. If we apply Lemma 5.3 to the antidiagonal $L_{a}$ in this monotone $S^{2} \times S^{2}$, we find a Lagrangian sphere in $\left(\mathbb{C} P^{2} \# 2 \overline{\mathbb{C P}}^{2}, \tau\right)$ in the class $E_{1}-E_{2}=\iota\left(\left[L_{a}\right]\right)$. In addition, such a Lagrangian sphere can be made disjoint from an embedded $H$-class sphere in $\mathbb{C} P^{2} \# 2 \overline{\mathbb{C P}}^{2}$ by Theorem 1.1. We therefore obtain a Lagrangian sphere in our local model.

In general, let $(M, \omega)$ be obtained by symplectically blowing down two disjoint spheres in $E_{1}$ and $E_{2}$ in $(\bar{M}, \bar{\omega})$ and adopt notation in Lemma 5.3. We shrink both balls corresponding to $E_{1}$ and $E_{2}$ to size $\epsilon \ll 1$. By the uniqueness of ball-embeddings (in case of absence of a Lagrangian sphere; see Remark 5.2), we may place the two tiny balls $V_{1}$ and $V_{2}$ in a Darboux chart. Our local model analysis above ensures that there is a Lagrangian sphere $L$ in the blow-up of the chart around $V_{1}$ and $V_{2}$. Consider the class $B_{b}=\operatorname{PD}\left(p^{*} \omega\right)-b E_{1}-b E_{2}$ where $b$ is a positive rational number slightly larger than $a=\bar{\omega}\left(E_{i}\right), i=1,2$. Since the $K_{\bar{\omega}}$-symplectic cone $\mathcal{C}_{K_{\bar{\omega}}}$ is open, we can further assume that $\operatorname{PD}\left(B_{b}\right)$ is in $\mathcal{C}_{K_{\bar{\omega}}}$. Clearly, $B_{b} \cdot\left(E_{1}-E_{2}\right)=0$. Thus for some large integer $l_{b}, l_{b} B_{b}$ satisfies the conditions in Lemma 5.1. Now the conclusion follows from inflating along a symplectic surface in class $B_{b}$ as in the proof of Lemma 5.3.

Corollary 5.5 Suppose $(M, \omega)$ is a minimal symplectic manifold with $b^{+}=1$, $[\omega] \in H^{2}(M, \mathbb{Q})$. Suppose $(\bar{M}, \bar{\omega})$ is a $k$ point symplectic blow-up of $(M, \omega)$ with $E_{i}$, $i=1, \ldots, k$, the corresponding exceptional class, and the canonical injective map is 
denoted as $\iota: H_{2}(M ; \mathbb{Z}) \rightarrow H_{2}(\bar{M} ; \mathbb{Z})$. Then $\xi \in H_{2}(\bar{M} ; \mathbb{Z})$ is a Lagrangian spherical class if

(1) either $\xi \in \operatorname{Im}(\iota)$ and $\iota^{-1}(\xi)$ is Lagrangian spherical,

(2) or $\xi=E_{i}-E_{j}$ for some $i, j$, ie $\xi$ is binary, and $\omega(\xi)=0$.

If $\kappa(M) \geq 0$, these are the only Lagrangian spherical classes of $(\bar{M}, \bar{\omega})$.

Proof (1) and (2) follow directly from Lemmas 5.3 and 5.4 respectively.

To show these are the only Lagrangian spherical classes when $\kappa(M) \geq 0$, suppose $\xi=\xi^{\prime}-\sum_{i=1}^{k} a_{i} E_{i}$ is represented by a Lagrangian sphere $\bar{L}$, where $\xi^{\prime} \in \operatorname{Im}(\iota)$.

If $a_{i}=0$ for all $i$, then apply Theorem 1.1 to find disjoint exceptional spheres in the classes $E_{i}$, which are also disjoint from $\bar{L}$. This shows that $\xi^{\prime}$ is a Lagrangian spherical class of $(M, \omega)$.

Now assume some $a_{i} \neq 0$. Without loss of generality, let $i=1$. The reflection $R(\xi)$ thus sends $E_{1}$ to $a \xi^{\prime}-\sum_{i>1} a_{i} E_{i}-\left(a_{1}^{2}-1\right) E_{1}$. Such a class is an exceptional class of $(\bar{M}, \bar{\omega})$. However, from the uniqueness of the minimal model for symplectic manifolds with $\kappa \geq 0$ [39], $a \xi^{\prime}-\sum_{i>1} a_{i} E_{i}-\left(a_{1}^{2}-1\right) E_{1}=E_{j}$ for some $j$. This shows $\xi^{\prime}=0$ and $\xi$ is indeed binary.

\subsubsection{Rational manifolds}

Proof of Theorem 1.4, rational manifold case The case of $S^{2} \times S^{2}$ is well-known and so we focus on blow-ups of $\mathbb{C} P^{2}$ below.

Due to the transitive action of $D(M)$ on $\mathcal{K}$ mentioned in Section 4 , and using Definition 4.12, we are reduced to prove the following Proposition.

Proposition 5.6 Suppose $M=\mathbb{C} P^{2} \# n \overline{\mathbb{C P}}^{2}$ with $\left\{H, E_{1}, \ldots, E_{n}\right\}$ a standard basis, and $\omega$ is a symplectic form with $K_{\omega}=K_{0}=\operatorname{PD}\left(-3 H+E_{1}+\cdots+E_{n}\right)$. Then $\xi \in H_{2}(M ; \mathbb{Z})$ is represented by a Lagrangian sphere if and only if $\xi$ is $\left(K_{0},[\omega]\right)-$ null spherical.

Proof The conditions are clearly necessary. In the case $n=2$, up to sign, the only $K_{0}$-null spherical class is the binary class $\xi=E_{1}-E_{2}$. And if $\xi$ is $\left(K_{0},[\omega]\right)-$ null spherical, then $E_{1}$ and $E_{2}$ must have equal symplectic area. Thus the existence of a Lagrangian sphere has been argued in the first paragraph of Lemma 5.4.

Let us then suppose that $n>3$. One notices that in this case $\xi$ can also be assumed to be binary. This is because, from Proposition 4.7, there is a self-diffeomorphism $\phi$ 
of $M$, which induces a $K_{0}$-twist on homology and sends $\xi$ to a binary class, and we could just consider $\phi_{*}(\xi)$ in $\left(M,\left(\phi^{-1}\right)^{*} \omega\right)$. Without loss of generality we could further assume $\xi=E_{1}-E_{2}$. If $\omega(\xi)=0$, then, up to scaling, $\operatorname{PD}([\omega])=3 H-\sum b_{i} E_{i}$ with $b_{1}=b_{2}=b>0$.

Blowing down a collection of disjoint exceptional spheres in the classes $E_{i}$ with $i \geq 3$, we obtain $M^{\prime}=\mathbb{C} P^{2} \# 2 \overline{\mathbb{C P}}^{2}$ with a symplectic form $\omega^{\prime}$ in the class $[\omega]=$ $\mathrm{PD}\left(3 H-b E_{1}-b E_{2}\right)$. As just shown, there is a Lagrangian sphere $L \subset\left(M^{\prime}, \omega^{\prime}\right)$ in the class $E_{1}-E_{2}$. Now apply Lemma 5.3 to obtain the desired Lagrangian sphere back in $(M, \omega)$ by performing $n-2$ blow-ups.

Finally let us suppose that $n=3$. A $K_{0}$-null spherical class is either binary or the ternary class $\xi=H-E_{1}-E_{2}-E_{3}$. The binary case can be treated in the same way as in the case $n>3$. So let us assume that $\xi=H-E_{1}-E_{2}-E_{3}$. Let $(\bar{M}, \bar{\omega})$ be a one point blow up of $(M, \omega), E_{4}$ the new exceptional class, and $\iota$ the canonical map. Notice that $b^{-}(\bar{M})=4$ and $\iota(\xi)$ is $\left(K_{0},[\bar{\omega}]\right)$-null spherical, thus there is a Lagrangian $\bar{L} \subset(\bar{M}, \bar{\omega})$ in the class $\iota(\xi)$. By applying Theorem 1.2 to $\bar{L}$ and $E_{4}$, we conclude the proof by blowing down an exceptional sphere in class $E_{4}$ disjoint from $\bar{L}$.

Now the proof of Theorem 1.4 in the rational manifold case is complete.

\subsubsection{Irrational ruled manifolds}

Proof of Theorem 1.4, irrational ruled manifold case Similar to the rational case, it reduces to the following statement.

Proposition 5.7 Suppose $M=\left(\Sigma_{h} \times S^{2}\right) \# n \overline{\mathbb{C P}}^{2}$ with $\left\{T, F, E_{1}, \ldots, E_{n}\right\}$ a standard basis, and $\omega$ is a symplectic form with $K_{\omega}=K_{0}=\operatorname{PD}\left(-2 T(2 h-2) F+E_{1}+\cdots+E_{n}\right)$. Then $\xi \in H_{2}(M ; \mathbb{Z})$ is represented by a Lagrangian sphere if and only if $\xi$ is $\left(K_{0},[\omega]\right)-$ null spherical.

Proof We use the cut and paste procedure in [39] to reduce it to the rational manifold case.

We can view $(M, \omega)$ as a symplectic genus 0 Lefschetz fibration over $\Sigma_{h}$ with $n$ reducible fibers, each consisting of a pair of exceptional spheres in the classes $E_{i}$ and $F-E_{i}$. Denote the projection by $\pi$ and the image of the reducible fibers by $B$. View $\Sigma_{h}$ as assembled from a $4 h$-sided polygon with the vertices going to $x_{0} \in \Sigma_{h}$, the edges going to a $2 h$-wedge of loops $\Lambda_{h}$. Since $B$ is a finite set, we can assume that $B \cap \Lambda_{h}=\varnothing$. 
We cut $M$ along $\pi^{-1}\left(\Lambda_{h}\right)$ to obtain a genus 0 Lefschetz fibration $V$ over a two-disk $D$ with $n$ reducible fibers. Recall from [39, Lemmas 4.13-4.14] that with a symplectic deformation supported near an arbitrarily small neighborhood of $x_{0},(M, \omega)$ can be assumed to be a symplectic product in a neighborhood of $\pi^{-1}\left(\Lambda_{h}\right)$. Therefore we can compactify $(V, \omega)$ into a genus 0 Lefschetz fibration $(\bar{V}, \bar{\omega})$ over $S^{2}$ with $n$ reducible fibers by adding a fiber $F_{0}$.

Notice that $V$ is diffeomorphic to $\left(S^{2} \times D^{2}\right) \# n \overline{\mathbb{C P}}^{2}$, and $\bar{V}$ is diffeomorphic to $\left(S^{2} \times S^{2}\right) \# n \overline{\mathbb{C P}}^{2}=\left(\mathbb{C} P^{2} \# \overline{\mathbb{C P}}^{2}\right) \# n \overline{\mathbb{C P}}^{2}$. Moreover, in the standard basis representation, $F$ corresponds to $H-E_{1}$, and $E_{i}$ corresponds to $E_{i}$. In particular, a $\left(K_{0},[\omega]\right)-$ null spherical class corresponds to either $H-E_{1}-E_{i}-E_{j}$ or $E_{i}-E_{j}, 2 \leq i<j \leq n$.

We have shown there are Lagrangian spheres in $(\bar{V}, \bar{\omega})$ in these classes. What remains to prove is that there are Lagrangian spheres disjoint from the symplectic sphere $F_{0}$. This is true due to Theorem 1.1 , since $\left[F_{0}\right]=H-E_{1}$ is a square 0 class, orthogonal to $H-E_{1}-E_{i}-E_{j}$ and $E_{i}-E_{j}$ for any $2 \leq i<j \leq n$.

\subsection{Homological action}

We are now ready to prove Theorem 1.8.

Proof Let $(M, \omega)$ be a symplectic 4 -manifold with $\kappa=-\infty$. Further assume that a standard basis is chosen. As mentioned in the proof of Theorem 1.4, fixing the canonical class causes no loss of generality. Thus we assume that $K_{\omega}=K_{0}$.

On the one hand, if $f \in \operatorname{Symp}(M, \omega)$, then $f_{*} \in D_{K_{0},[\omega]}(M)$. On the other hand, Theorem 1.4 implies any $\left(K_{0},[\omega]\right)$-twist is realized by a Lagrangian Dehn twist. With this understood, Theorem 1.8 is simply a consequence of Proposition 4.14, Lemma 4.16, and Theorem 1.4.

Corollary 5.8 If $(M, \omega)$ is monotone, the representation of the symplectic mapping class group on $H_{2}(M ; \mathbb{Z})$, namely, the Torelli part, is $D_{K_{\omega}}(M)$.

Remark 5.9 Corollary 5.5 also has its counterpart asserting when $b^{+}(M)=1$ and $\kappa(M) \geq 0$, the homological action of $\operatorname{Symp}(\bar{M}, \bar{\omega})$ is generated by the homological action of $\operatorname{Symp}(M, \omega)$ and binary Lagrangian reflections.

It would be interesting to know whether for any minimal $(M, \omega)$ with $b^{+}=1$ and $\kappa(M) \geq 0$, the homological action of $\operatorname{Symp}(M, \omega)$ is generated by Lagrangian Dehn twists. 


\section{Uniqueness of Lagrangian spheres in rational manifolds}

The present section is devoted to the proof of Theorem 1.5. We begin by reviewing two basic uniqueness results of Hind for $S^{2} \times S^{2}$ and $T^{*} S^{2}$.

\subsection{Review of Hind's results}

6.1.1 $S^{2} \times S^{2}$ via symplectic cut For $S^{2} \times S^{2}$ we have uniqueness up to isotopy:

Theorem 6.1 (Hind [22]) Lagrangian spheres in a monotone $S^{2} \times S^{2}$ are unique up to Hamiltonian isotopy.

From the connectedness of $\operatorname{Symp}\left(S^{2} \times S^{2}, \sigma \oplus \sigma\right)$ by Gromov [20], Theorem 6.1 is equivalent to:

Proposition 6.2 Lagrangian spheres in a monotone $S^{2} \times S^{2}$ are unique up to symplectomorphisms.

We here offer an argument for this weaker version of uniqueness using an idea from Hind [21] turning the Lagrangian uniqueness problem into a symplectic uniqueness problem via symplectic cut. Such an argument is useful for the uniqueness of Lagrangian $\mathbb{R} P^{2}$ in rational manifolds (see Section 6.4.1). Some preparations are needed.

Let $A, B \in H_{2}\left(S^{2} \times S^{2} ; \mathbb{Z}\right)$ be the classes of two product factors on $S^{2} \times S^{2}$. Let $\Omega_{\lambda}$ be the product symplectic form $\pi_{1}^{*} \sigma+(1+\lambda) \pi_{2}^{*} \sigma$ with $\lambda>0$. Let $\mathcal{J}_{\lambda}$ be the space of $\Omega_{\lambda}$-tamed almost complex structures. The following is due to Abreu and McDuff:

Theorem 6.3 [1, Proposition 2.1, Corollary 2.8] Suppose $l-1<\lambda \leq l, l$ an integer. Then $\mathcal{J}_{\lambda}$ admits a stratification $\left\{U_{k}\right\}_{0 \leq k \leq l}$ with the following properties:

(1) The class $A-k B$ is represented by a unique embedded $J$-holomorphic sphere if and only if $J \in U_{k}$.

(2) Each $U_{k}$ is connected.

As a consequence, we have the following claim:

Proposition 6.4 The space of symplectic spheres with self-intersection $-2 k$ in $\left(S^{2} \times S^{2}, \omega_{\lambda}\right)$ is nonempty and connected if $\lambda>k-1$. 
Proof A symplectic sphere with self-intersection $-2 k$ is in the class $A-k B$, and it exists if and only if $\lambda>k-1$. For two such symplectic spheres $C_{i}, i=0,1$, there are almost complex structures $J_{i} \in U_{k}$ such that $C_{i}$ is $J_{i}$-holomorphic for $i=0,1$. By Theorem 6.3(2), there is a path $J_{t}$ in $U_{k}$ connecting $J_{0}$ and $J_{1}$. By Theorem 6.3(1), there is a unique sphere $C_{t}$ with self-intersection $-2 k$ for each $J_{t}$. This path of symplectic spheres is continuous due to Gromov's compactness.

Proof of Proposition 6.2 Given two Lagrangian spheres $L_{1}, L_{2}$ in $S^{2} \times S^{2}$ with a monotone symplectic form $\omega$. By Weinstein's neighborhood theorem one can fix two symplectic embeddings $\phi_{1}, \phi_{2}: T_{r}^{*} S^{2} \rightarrow S^{2} \times S^{2}$ for some small $r>0$. For each $i$, consider the geodesic flow on $S^{2}$ with the standard round metric. By performing symplectic cut on $\left(S^{2} \times S^{2}, \omega\right)$ along the boundary of the image of $\phi_{i}$, we obtain a pair of $S^{2} \times S^{2}$ for each $i$ : one comes from $\phi_{i}\left(T_{r}^{*} S^{2}\right)$, equipped with the standard monotone symplectic form of size $r$; and the other one comes from the complement of $\phi_{i}\left(T_{r}^{*} S^{2}\right)$, equipped with symplectic form $\omega_{i}$ and a symplectic $(-2)$-sphere $\Sigma_{i}$. Clearly, for the $S^{2} \times S^{2}$ coming from the complement of $\phi_{i}\left(T_{r}^{*} S^{2}\right)$, one may find a diffeomorphism $\tilde{\iota}$ between them, so that $\left[\tilde{\iota}^{*} \omega_{2}\right]=\left[\omega_{1}\right]$.

It follows from the uniqueness of cohomologous symplectic structures in [29] and Proposition 6.4, we can upgrade $\tau$ to the following symplectomorphism of pairs, by composing an appropriate diffeomorphism

$$
\iota:\left(\left(S^{2} \times S^{2}, \omega_{1}\right), \Sigma_{1}\right) \rightarrow\left(\left(S^{2} \times S^{2}, \omega_{2}\right), \Sigma_{2}\right),
$$

where $\iota$ sends a neighborhood of $\Sigma_{1}$ symplectomorphically to one of $\Sigma_{2}$. Notice the following useful fact: the symplectic sum [19] is the exact inverse of symplectic cut. Here we make this precise by providing a general description of symplectic sum pointed out to us by Gompf.

Let $(M, \Sigma)$ and $\left(N, \Sigma^{\prime}\right)$ be two symplectic pairs, where $\Sigma$ and $\Sigma^{\prime}$ are symplectomorphic, whose normal bundles have opposite Euler classes. Let $P$ be the (real) projectivization of one of the normal bundles, then for some interval $(-a, a), P \times(-a, a)$ has a canonical symplectic form with Hamiltonian $S^{1}$-action rotating each fiber. The symplectic cut on $P \times(-a, a)$ at 0 gives two disc bundles on $\Sigma$ and $\Sigma^{\prime}$ with opposite Euler classes as they are embedded in $M$ and $N$, respectively. Therefore, the complement of $P \times\{0\}$ can be locally identified with the two normal bundles of $\Sigma$ and $\Sigma^{\prime}$ removing the zero section. Such a gluing completes the symplectic sum operation.

With this understood, we can glue $\iota$ with the identity symplectomorphism on the monotone $S^{2} \times S^{2}$ with size $r$, up to an appropriate adjustment on $\iota$. Explicitly, when performing the pair of symplectic cuts, there is associated a natural identification between some neighborhoods $U_{i}$ of $\Sigma_{i}$ for $i=1,2$, as described above. The map $\iota$ 
cannot be glued directly to the identity map on the other piece, precisely because it is not the identity map when represented under such an identification between the $U_{i}$ 's.

Therefore, our question is local, and in below, by shrinking $U_{1}$ if necessary, we regard $U_{1}$ a subset of $U_{2}$ using the natural identification. We further assume $\iota\left(U_{1}\right) \subset U_{2}$.

Notice that $\Sigma_{1}$ and $\Sigma_{2}$ are naturally parameterized by $S^{2}$ via $\phi_{1}$ and $\phi_{2}$. With respect to these parameterizations, $\iota$ restricted to $\Sigma_{1}$ may not be the identity map. However, it is clear that there is a self-symplectomorphism $\tilde{f}$ of $S^{2} \times S^{2}$ with respect to $\omega_{2}$ so that $\tilde{f} \circ \iota$ is the identity on the zero section by a standard application of Moser's argument. Therefore, without loss of generality, we simply assume $\left.\iota\right|_{\Sigma_{1}}$ is just the identity.

Now it suffices for us to show that, by composing a certain self-symplectomorphism $f$ of $S^{2} \times S^{2}$ with respect to $\omega_{2}$, which is the identity on $\Sigma_{2}$ and supported in $U_{1} \cap \iota\left(U_{1}\right)$, so that $f \circ \iota$ is the identity near $\Sigma_{1}$ under the above identification. Furthermore, this is equivalent to finding an $f$ so that $f \circ \iota$ is the identity on the normal bundle of $\Sigma_{1}$ (see [11;24], etc.). In the rest of the proof we pursue such an $f$.

To achieve this we embed $U_{2}$ symplectically into $\mathcal{O}(-2)$. Here $\mathcal{O}(n)$ denotes the complex line bundle over $S^{2}$ with $c_{1}=n$, whose total space is equipped with a standard Kähler form, agreeing with $\omega_{2}$ on $\Sigma_{2}$ when restricted to the zero section.

Denote $d \iota$ as the induced tangent map on $\left.T\left(S^{2} \times S^{2}\right)\right|_{\Sigma_{1}}$, our assertion is equivalent to finding a symplectomorphism $f$ of $\mathcal{O}(-2)$ so that $d f=(d \iota)^{-1}$ and $\operatorname{supp}(f) \subset$ $U_{1} \cap \iota\left(U_{1}\right)$. Now the assertion follows from [24, Lemma 2.4]. To recall the lemma, let $\mathcal{G}_{s}$ be the symplectic gauge transformations of the normal bundle $v$ of $\Sigma_{2}$, that is, sections of $\operatorname{Sp}(\nu) \backslash \Sigma_{2}$, where $\operatorname{Sp}(v)$ are the fiberwise symplectic linear maps. Notice that $\mathcal{G}_{s}=\operatorname{Map}\left(\Sigma_{2} ; \operatorname{SL}(2 ; \mathbb{R})\right) \simeq S^{1}$. Let $S_{c}(\mathcal{O}(n))_{0}$ be the compactly supported symplectomorphism fixing the zero section.

Lemma 6.5 [24, Lemma 2.4] The homomorphism $S_{c}(\mathcal{O}(n)) \rightarrow \mathcal{G}_{s}$ given by taking derivatives along $\Sigma$ is surjective.

Notice that [24] assumes $n \geq 0$ throughout, but the proof of Lemma 6.5 indeed works for arbitrary $n \in \mathbb{Z}$. Given $(d \iota)^{-1} \in \mathcal{G}_{S}$, from the homotopy type of $\mathcal{G}_{s}$, we can connect to it by a path $\gamma(t), t \in[0,1]$ starting from id. Take a lift $\tilde{\gamma}$ of $\gamma$ by Lemma 6.5 starting from id $\in S_{c}(\mathcal{O}(-2))_{0}$. Since $\mathcal{O}(-2)$ is simply connected, $\tilde{\gamma}(t)$ are Hamiltonian isotopies, hence their supports can be cut-off to fit inside $U_{1} \cap \iota\left(U_{1}\right)$ without affecting the action restricting to $\left.T(\mathcal{O}(-2))\right|_{\Sigma_{2}}$. In particular, since the support of $\tilde{\gamma}(1)$ is contained in $U_{1} \cap \iota\left(U_{1}\right)$, it can be viewed as a self-symplectomorphism of $S^{2} \times S^{2}$ with respect to $\omega_{2}$. Thus $\tilde{\gamma}(1)$ is the symplectomorphism so that $f=\tilde{\gamma}(1) \circ \tilde{f}$ has the desired property. This in turn concludes our proof of Proposition 6.2. 
6.1.2 $T^{*} S^{2}$ and the symplectic mapping class group Further exploring the symplectic cut approach in Section 6.1.1, we obtain an alternative proof of Hind's Lagrangian sphere uniqueness in $T^{*} S^{2}$ below via Seidel's description of the compactly supported symplectomorphism group of $T^{*} S^{2}$.

Theorem 6.6 (Hind [21]) Lagrangian spheres in $\left(T^{*} S^{2}, \omega_{\text {std }}\right)$ are unique up to Hamiltonian isotopy.

Proof Via the negative Liouville flow and scaling we can isotope any Lagrangian in $\left(T^{*} S^{2}, \omega_{\text {std }}\right)$ into one in $\left(T_{1}^{*} S^{2}, \omega_{\text {std }}\right)$. Further, via the identification $\left(T_{1}^{*} S^{2}, \omega_{\text {std }}\right)=$ $\left(S^{2} \times S^{2}, \omega_{0}\right) \backslash \Delta$, where $\omega_{0}$ is a monotone form and $\Delta$ is the diagonal of $S^{2} \times S^{2}$, it suffices to show the uniqueness of Lagrangian spheres in $\left(S^{2} \times S^{2}, \omega_{0}\right) \backslash \Delta$.

Given two Lagrangian spheres $L_{1}, L_{2} \in\left(S^{2} \times S^{2}, \omega_{0}\right) \backslash \Delta$, we first claim that there is $\phi \in \operatorname{Symp}_{c}\left(T_{1}^{*} S^{2}, \omega_{\text {std }}\right)$ such that $\phi\left(L_{1}\right)=L_{2}$, where $\operatorname{Symp}_{c}$ denotes the compactly supported symplectomorphism group.

Without loss of generality we assume $L_{2}=\bar{\Delta}$, which is the antidiagonal, corresponding in turn to the zero section of $T^{*} S^{2}$. By Proposition 6.2, there is $\Psi \in$ $\operatorname{Symp}\left(S^{2} \times S^{2}, \omega_{0}\right)$, such that $\Psi\left(L_{1}\right)=L_{2}$. $\Psi$ may not fix $\Delta$, but notice that $\Psi(\Delta) \cap \bar{\Delta}\left(=L_{2}\right)=\varnothing$. Since the complement of $\bar{\Delta}$ is canonically identified with a symplectic disk bundle over the diagonal, by [23] there is a symplectic isotopy $\widetilde{\Phi}_{t}: S^{2} \rightarrow\left(S^{2} \times S^{2}, \omega_{0}\right)$ fixing $\bar{\Delta}$ and connecting the two symplectic spheres $\Psi(\Delta)$ and $\Delta$. In particular, $\widetilde{\Phi}_{t} \circ \Psi(\Delta)$ is disjoint from $\bar{\Delta}$ for each $t$.

Now we extend $\widetilde{\Phi}_{t}$ to a symplectic isotopy of a neighborhood $U$ of $\Psi(\Delta)$ which we still denote as $\widetilde{\Phi}_{t}$ [41, Example 3.40], and require that $\widetilde{\Phi}_{t}(U)$ be still disjoint from $\bar{\Delta}$ for all $t$. We then trivially extend $\widetilde{\Psi}_{t}$ to $\widetilde{\phi}_{t}$, a symplectic isotopy on a neighborhood $U^{\prime}$ of $\Psi(\Delta) \cup \bar{\Delta}$, which restricts to $\widetilde{\Phi}_{t}$ on $U$ and to the identity near $\bar{\Delta}$. Since $H^{1}\left(U^{\prime} ; \mathbb{R}\right)=0, H^{2}\left(S^{2} \times S^{2}, U^{\prime} ; \mathbb{R}\right)$ injects into $H^{2}\left(S^{2} \times S^{2} ; \mathbb{R}\right)$. By the argument proving Banyaga's isotopy extension theorem (see for example [41, Theorem 3.19]), $\widetilde{\phi}_{t}$ extends to a global symplectic isotopy $\phi_{t}$ of $\left(S^{2} \times S^{2}, \omega_{0}\right)$, where $\phi_{0}=\mathrm{id}$, $\phi_{1}\left(L_{1}\right)=L_{2}$, and $\left.\phi_{1}\right|_{\Delta}=\mathrm{id}$.

Consider $\phi^{\prime}=\phi_{1} \circ \Psi \in \operatorname{Symp}\left(S^{2} \times S^{2}, \omega_{0}\right)$. Since $\phi^{\prime}$ is the identity on $\Delta$, it induces a compactly supported symplectomorphism $\phi$ of $\left(T_{1}^{*} S^{2}, \omega_{\text {std }}\right)$ up to isotopy, mapping $L_{1}$ to the zero section $L_{2}$.

From Seidel's description of $\operatorname{Symp}_{c}\left(T_{1}^{*} S^{2}, \omega_{\text {std }}\right)$ in [46], $\phi=\tau^{n} \circ \eta_{1}$, where $\tau$ is the Lagrangian Dehn twist along the zero section $L_{2}$, and $\eta_{t}, t \in[0,1]$ with $\eta_{0}=$ id is a compactly supported symplectic isotopy. Now it is clear that $\eta_{t}\left(L_{1}\right)$ is a path connecting $L_{1}$ to the zero section since $\tau$ fixes the zero section. 


\subsection{Proof of Theorem 1.5}

For $k \geq 0$ we will denote by $V_{k}$ the manifold $\left(S^{2} \times S^{2}\right) \# k \overline{\mathbb{C P}}^{2}$. When $k \geq 1$, $V_{k}=\mathbb{C} P^{2} \#(k+1) \overline{\mathbb{C P}}^{2}$. Due to Theorem 6.1 and the fact that $\mathbb{C} P^{2} \# \overline{\mathbb{C P}}^{2}$ has no spheres with self-intersection -2 , we only need to prove Theorem 1.5 for $V_{k}$ with $k=1,3$, and $k=2$ but $[L]$ not characteristic. By Proposition 4.10, we may further assume that $[L]$ is the binary class $E_{1}-E_{2}$.

Throughout this subsection, $J_{0}$ denotes the complex structure obtained from a generic $k$-point complex blow-up of $\mathbb{C} P^{1} \times \mathbb{C} P^{1}$. Without loss of generality, we may assume $\omega$ is a Kähler form compatible with $J_{0}$. This follows from [34, Proposition 4.8] that the symplectic cone is the same as the $J_{0}$-compatible cone in $H^{2}\left(V_{k}, \mathbb{R}\right)$ when $k \leq 8$, as well as the uniqueness of homologous symplectic forms in [40].

To prove Theorem 1.5, we apply Theorem 1.1 and follow the approach of Evans [13] where the monotone case is settled. For some of the details one is referred to of Evans [13, Section 9; 14, 4.2].

For the binary class $E_{1}-E_{2}$, the following stable symplectic sphere configuration type (Definition 3.3) $D_{E_{1}-E_{2}}$ is introduced in [13]:

- $\left\{H-E_{1}-E_{2}, H\right\}$ when $k=1$,

- $\left\{H-E_{1}-E_{2}, H-E_{3}, E_{3}\right\}$ when $k=2$,

- $\left\{H-E_{1}-E_{2}, H-E_{3}-E_{4}, E_{3}, E_{4}\right\}$ when $k=3$.

Since $\left(V_{k}, J_{0}\right)$ is a generic blow up, it is clear that there is a $J_{0}$-holomorphic $D_{E_{1}-E_{2}}$ configuration $C_{0}$.

Lemma 6.7 Suppose $L$ is a Lagrangian sphere in $\left(V_{k}, \omega\right)$ with $k \leq 3$ and $[L]=$ $E_{1}-E_{2}$. Then $L$ can be Hamiltonian isotoped off $C_{0}$.

Proof From Corollary 3.13, in the complement of the given Lagrangian sphere $L$, we can find a $D_{E_{1}-E_{2}}$-configuration $C$.

By Proposition 3.4, $C_{0}$ and $C$ are symplectically isotopic. Following the proof of [14, Theorem 9], with a small perturbation along the isotopy, we may assume the symplectic spheres in the configuration intersect $\omega$-orthogonally during the isotopy. Thus, by the symplectic neighborhood theorem, we can extend this isotopy to a neighborhood of the configuration. From the fact that $C$ and $C_{0}$ have trivial $H^{1}$, as in the proof of Theorem 6.6, we obtain an ambient Hamiltonian isotopy $\Psi_{t}$ taking $C$ to $C_{0}$. In particular, $L$ is Hamiltonian isotopic to $\Psi_{1}(L)$ which is disjoint from $C_{0}$. 
Proposition 6.8 Suppose there is a Lagrangian sphere $L$ in $\left(V_{k}, \omega\right)$ with $k \leq 3$ and $[L]=E_{1}-E_{2}$. When $[\omega]$ is a rational, the complement of $C_{0}$ contains a unique Lagrangian sphere up to Lagrangian isotopy.

Proof By Lemma 6.7 we can assume that the Lagrangian sphere $L$ is in the complement of $C_{0}$, so the complement of $C_{0}$ contains at least one Lagrangian sphere.

We will discuss the case $k=3$. The cases $k=1,2$ are similar. Up to scaling, we can write $\operatorname{PD}([\omega])=a H-E_{1}-E_{2}-b_{3} E_{3}-b_{4} E_{4}$ since $\omega([L])=0$. Further, $a>1+b_{i}$ since $\omega\left(H-E_{1}-E_{i}\right)>0$ for $i=3,4$. Rewrite

$\mathrm{PD}([\omega])=\left(H-E_{1}-E_{2}\right)+(a-1)\left(H-E_{3}-E_{4}\right)+\left(a-1-b_{3}\right) E_{3}+\left(a-1-b_{4}\right) E_{4}$.

Notice that $a, b_{i} \in \mathbb{Q}^{+}$since $[\omega]$ is assumed to rational. Since all coefficients are rational and positive, there is a large integer $l$, such that $\operatorname{PD}([l \omega])$ is represented as an positive integral combination of $\left\{H-E_{1}-E_{2}, H-E_{3}-E_{4}, E_{3}, E_{4}\right\}$, say, with coefficients $u, v, w, z \in \mathbb{Z}^{+}$.

If $C_{0}=C_{H-E_{1}-E_{2}} \cup C_{H-E_{3}-E_{4}} \cup C_{E_{3}} \cup C_{E_{4}}$, consider the divisor

$$
F=u C_{H-E_{1}-E_{2}}+v C_{H-E_{3}-E_{4}}+w C_{E_{3}}+z C_{E_{4}} .
$$

There is a holomorphic line bundle $\mathcal{L}$ with a holomorphic section $s$ whose zero divisor is exactly $F$. Take an hermitian metric and a compatible connection on $\mathcal{L}$ such that the curvature form is just $l \omega . \phi=-\log |s|^{2}$ defines a plurisubharmonic function with $-d\left(d \phi \circ J_{0}\right)=l \omega$ on the complement $U_{0}$ of the $C_{0}$.

Notice that $U_{0}$ is the same as the complement $U$ in [14, Proposition 4.2.1], which is shown to be biholomorphic to the affine quadric there. The rest of the argument is exactly as in the proof of [14, Proposition 4.2.1], reducing to Theorem 6.6, the uniqueness in $\left(T^{*} S^{2}, \omega_{\mathrm{std}}\right)$.

Consider the finite type Stein structure $\left(J_{0}, \phi / l\right)$ on $U_{0}$. Define $h: \mathbb{R} \rightarrow \mathbb{R}$ to be the function $h(x)=e^{x}-1$ and $\phi_{h}=h \circ \phi$. By [7, Lemma 3.1; [48, Lemma 6]], $\left(U_{0}, J_{0}, \phi_{h}\right)$ is a complete Stein manifold of finite type with Kähler form $\omega_{h}=-d\left(d \phi_{h} \circ J_{0}\right)$. Suppose a sublevel set $Y=\phi^{-1}[0, k]$ contains all the critical points of $\phi$. View $(Y, \omega)$ as a Liouville domain, and let $(\hat{Y}, \widehat{\omega})$ be its symplectic completion. By [14, Lemma 2.1.5], $\left(U_{0}, \omega_{h}\right)$ is symplectomorphic to $(\hat{Y}, \widehat{\omega})$.

Since the affine quadric $Q$ has a complete finite type Stein structure inherited from $\mathbb{C}^{3}$, it follows from [14, Lemma 2.1.6] that $\left(U_{0}, \omega_{h}\right)$ is symplectomorphic to $\left(Q, \omega_{\text {can }}\right)$. Combining all the symplectomorphisms, we find that the Liouville manifold $(\widehat{Y}, \widehat{\omega})$ is symplectomorphic to $\left(T^{*} S^{2}, \omega_{\text {std }}\right)$. 
Given any two Lagrangian spheres $L_{0}, L_{1}$ in the complement of $C_{0}$, they lie in a sublevel set $Y$ of $\phi$ containing all the critical points. We obtain an isotopy $L_{t}$ in $(\hat{Y}, \widehat{\omega})$ by Hind's Theorem 6.6. Contract the isotopy $L_{t}$ into the sublevel set $Y$ using the negative Liouville flow on $(\hat{Y}, \widehat{\omega})$. The endpoints of the contracted isotopy are also connected in $Y$ to $L_{0}$ and $L_{1}$ respectively by the positive Liouville flow. Therefore, one gets the desired Hamiltonian isotopy between $L_{0}$ and $L_{1}$ in $Y \subset U_{0}$.

Proof of Theorem 1.5 As mentioned in the beginning of this subsection, we could assume that $M=V_{k}$ with $k=1,2,3, \omega$ is a Kähler form compatible with $J_{0}$, and $\xi=E_{1}-E_{2}$.

Suppose $L_{0}$ and $L_{1}$ are two Lagrangian spheres in the class $\xi$. By Lemma 6.7 they are Hamiltonian isotopic respectively to two Lagrangian spheres, still denoted by $L_{0}$ and $L_{1}$, in the complement $U_{0}$ of $C_{0}$. We will show that $L_{0}$ and $L_{1}$ are Lagrangian isotopic in $U_{0}$, and hence in $\left(V_{k}, \omega\right)$. As argued in Theorem 6.6, this implies that $L_{0}$ and $L_{1}$ are Hamiltonian isotopic.

Again we will discuss the case $k=3$. Notice that the $\omega$-area of $E_{1}$ and that of $E_{2}$ are the same; by rescaling the symplectic form, we could assume the $\omega$-area of $E_{i}$, $i=1,2$ is rational. View $\left(V_{3}, \omega\right)$ as a three point blow-up of a monotone $\left(S^{2} \times S^{2}, \tau\right)$,

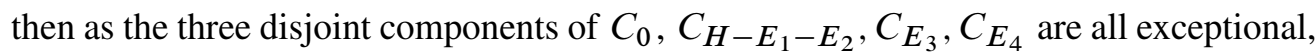
corresponding to three ball embeddings $h_{12}, e_{3}, e_{4}$ in $\left(S^{2} \times S^{2}, \tau\right)$. Let $\widetilde{L}_{0}$ and $\widetilde{L}_{1}$ be the corresponding Lagrangians in $\left(S^{2} \times S^{2}, \tau\right)$.

Via the correspondence of ball-embeddings and symplectic forms in the blown-up manifolds, one may deform $\omega$ to $\omega^{\prime}$ near $C_{H-E_{1}-E_{2}}, C_{E_{3}}, C_{E_{4}}$ such that their $\omega^{\prime}$-areas become rational. In fact, from the continuity of ball embeddings, such a deformation can be chosen to correspond to a slightly larger ball-embeddings $h_{12}^{\prime}, e_{3}^{\prime}$ and $e_{4}^{\prime}$ in $\left(S^{2} \times S^{2}, \tau\right)$. Further, we may assume that the larger embedded balls are still disjoint from $\widetilde{L}_{0}$ and $\widetilde{L}_{1}$. And when such a perturbation is chosen small enough, $J_{0}$ is still tamed by $\omega^{\prime}$ so that the configuration $C_{0}$ is still symplectic with respect to $\omega^{\prime}$.

Notice that $L_{0}$ and $L_{1}$ remain Lagrangian in $\left(V_{k}, \omega^{\prime}\right)$. Notice also that $\left[\omega^{\prime}\right]$ is rational, so we have a Lagrangian isotopy between $L_{0}$ and $L_{1}$ in $\left(V_{3}, \omega^{\prime}\right)$ by Proposition 6.8. It is important to observe that such an isotopy can be chosen to lie inside the complement of the $\omega^{\prime}$-symplectic configuration $C_{0}$.

In particular, the isotopy does not intersect the spheres $C_{H-E_{1}-E_{2}}, C_{E_{3}}, C_{E_{4}}$. In turn it gives rise to an isotopy between $\widetilde{L}_{0}$ and $\widetilde{L}_{1}$ in the complement of the images of $h_{12}^{\prime}, e_{3}^{\prime}$ and $e_{4}^{\prime}$. Since $h_{12}^{\prime}, e_{3}^{\prime}$ and $e_{4}^{\prime}$ are extensions of $h_{12}, e_{3}$ and $e_{4}$, the isotopy between $\widetilde{L}_{0}$ and $\widetilde{L}_{1}$ lie in the complement of the images of $h_{12}, e_{3}$ and $e_{4}$. Therefore it gives rise to an isotopy between $L_{0}$ and $L_{1}$ in the complement of the spheres $C_{H-E_{1}-E_{2}}, C_{E_{3}}, C_{E_{4}}$ in $\left(V_{k}, \omega\right)$. 


\subsection{Smooth isotopy}

Proof of Theorem 1.6 By Proposition 4.10, we again assume that we are in the binary case $E_{1}-E_{2}$. Given two Lagrangian spheres $L_{i}$, following [14], consider the classes $E_{j}, j \geq 3$. From Theorem 1.1, for each $i$, we can find a set of disjoint symplectic spheres in $E_{j}$, which are also disjoint from $L_{i}$. Applying Proposition 3.4 to these two stable spherical symplectic configurations as above, we can assume that $L_{i}$ are both disjoint from a set of disjoint symplectic spheres $S_{i}$ in $E_{j}, j \geq 3$.

Blow down $S_{i}$ we obtain $\left(\mathbb{C} P^{2} \# 2 \overline{\mathbb{C P}}^{2}, \omega^{\prime}\right)$ with balls $B_{j}$ disjoint from $L_{i}$. Let $L_{t}$ be a Lagrangian isotopy between $L_{i}$ in $\left(\mathbb{C} P^{2} \# 2 \overline{\mathbb{C P}}^{2}, \omega\right)$ from Theorem 1.5. Viewed as a smooth isotopy, we can assume that $L_{t}$ is transversal to the centers $x_{j}$ of $B_{j}$, thus avoiding $x_{j}$. Let $B_{j}^{\prime} \subset B_{j}$ be a smaller ball not intersecting $L_{t}$. Let $\phi$ be a diffeomorphism from $U^{\prime}$, the complement of $\bigcup B_{j}^{\prime}$ to $U$, the complement of $\bigcup B_{j}$, which is identity near $L_{i}$. Then $\phi\left(L_{t}\right)$ is a smooth isotopy between $L_{i}$ in $U$. Blowing up at $x_{j}$ by cutting $B_{j}$, we get back to $(M, \omega)$ and a smooth isotopy between $L_{i}$ therein.

\subsection{Some remarks on uniqueness}

We end the paper with some discussions about uniqueness.

6.4.1 Lagrangian $\mathbb{R} P^{2}$ The argument in Section 6.1.1, with (-2)-spheres replaced by $(-4)$-spheres, can be used to prove that any two Lagrangian $\mathbb{R} P^{2}$ in $\left(\mathbb{C} P^{2}, \omega_{\text {std }}\right)$ are symplectomorphic. From Gromov's connectedness of $\operatorname{Symp}\left(\mathbb{C} P^{2}, \omega_{\text {std }}\right)$ in [20], we then obtain a new proof of the following result of Hind [21].

Theorem 6.9 (Hind) Any two Lagrangian $\mathbb{R} P^{2}$ in $\mathbb{C} P^{2}$ are Hamiltonian isotopic to each other.

6.4.2 Uniqueness up to symplectomorphisms Conjecture 1.7 states that, for any two homologous Lagrangian spheres $L_{1}$ and $L_{2}$ in a symplectic rational manifold $(M, \omega)$, there exists $\phi \in \operatorname{Symp}_{h}(M, \omega)$ such that $\phi\left(L_{1}\right)=L_{2}$. It implies the disconnectedness of homologically trivial symplectomorphism groups in the cases when there are nonisotopic Lagrangian spheres.

We outline a possible approach to Conjecture 1.7. One easily reduces the problem to the binary case as in the proof of Theorem 1.4. Without loss of generality, let $\left[L_{i}\right]=E_{1}-E_{2}$.

For each pair $\left(M, L_{i}\right)$, by Theorem 1.1, away from $L_{i}$, there is a set of disjoint (-1) symplectic spheres $C_{i}^{l}, l=3, \ldots, k+1$, with $\left[C_{i}^{l}\right]=E_{l}$ for $l=3, \ldots, k$, 
and $\left[C_{i}^{k+1}\right]=H-E_{1}-E_{2}$. Blowing down the $C_{l}$ yields two $(k+1)$-tuples of $\left(\widetilde{M}_{i}, \widetilde{L}_{i}, B_{i}^{l}\right), i=1,2,3 \leq l \leq k+1$. Here $\widetilde{M}_{i}$ is a symplectic $S^{2} \times S^{2}, \widetilde{L}_{i}$ a Lagrangian sphere, and $B_{i}^{l}$ a symplectic ball corresponding to $C_{i}^{l}$.

By [29] there is a symplectomorphism $\Psi: \widetilde{M}_{1} \rightarrow \widetilde{M}_{2}$. From Proposition 6.2, there is a symplectomorphism sending $\Psi\left(\widetilde{L}_{1}\right)$ to $\widetilde{L}_{2}$. Composing these two symplectomorphisms one obtains a symplectomorphism between the pairs $\left(\widetilde{M}_{i}, \widetilde{L}_{i}\right)$, which we still denote as $\Psi$. The conjectured connectedness of relative symplectic ball embedding in Remark 5.2 implies that the $k-2$ balls $\Psi\left(B_{1}^{l}\right)$ can be further displaced by an $\tilde{L}_{2}$-preserving Hamiltonian isotopy to the balls $B_{2}^{l}$. This gives a symplectomorphism between the $(k+1)$-tuples $\left(\widetilde{M}_{i}, \widetilde{L}_{i}, B_{i}^{l}\right)$, which in turn descends to a symplectomorphism between the pairs $\left(M, L_{i}\right)$.

\section{References}

[1] M Abreu, D McDuff, Topology of symplectomorphism groups of rational ruled surfaces, J. Amer. Math. Soc. 13 (2000) 971-1009 MR1775741

[2] M Alberich-Carramiñana, Geometry of the plane Cremona maps, Lecture Notes in Math. 1769, Springer, Berlin (2002) MR1874328

[3] M Audin, Lagrangian skeletons, periodic geodesic flows and symplectic cuttings, Manuscripta Math. 124 (2007) 533-550 MR2357797

[4] J-F Barraud, Nodal symplectic spheres in $\mathbf{C P}^{2}$ with positive self-intersection, Internat. Math. Res. Notices (1999) 495-508 MR1692591

[5] P Biran, Symplectic packing in dimension 4, Geom. Funct. Anal. 7 (1997) 420-437 MR1466333

[6] P Biran, A stability property of symplectic packing, Invent. Math. 136 (1999) 123-155 MR1681101

[7] P Biran, K Cieliebak, Symplectic topology on subcritical manifolds, Comment. Math. Helv. 76 (2001) 712-753 MR1881704

[8] P Biran, O Cornea, Quantum Structures for Lagrangian submanifolds arXiv: 0708.4221

[9] F Bourgeois, A Morse-Bott approach to contact homology, $\mathrm{PhD}$ thesis, New York University (2002) Available at http://homepages.ulb.ac.be/ fbourgeo/pspdf/ thesis.pdf

[10] F Bourgeois, Y Eliashberg, H Hofer, K Wysocki, E Zehnder, Compactness results in symplectic field theory, Geom. Topol. 7 (2003) 799-888 MR2026549

[11] J Coffey, Symplectomorphism groups and isotropic skeletons, Geom. Topol. 9 (2005) 935-970 MR2140995 
[12] C Conley, E Zehnder, Morse-type index theory for flows and periodic solutions for Hamiltonian equations, Comm. Pure Appl. Math. 37 (1984) 207-253 MR733717

[13] J D Evans, Lagrangian spheres in del Pezzo surfaces, J. Topol. 3 (2010) 181-227 MR2608481

[14] J D Evans, Symplectic topology of some Stein and rational surfaces, $\mathrm{PhD}$ thesis, University of Cambridge (2010) Available at http://www.math.ethz.ch/ evansj/ thesis.pdf

[15] J D Evans, Symplectic mapping class groups of some Stein and rational surfaces, J. Symplectic Geom. 9 (2011) 45-82 MR2787361

[16] R Fintushel, R J Stern, Invariants for Lagrangian tori, Geom. Topol. 8 (2004) 947-968 MR2087074

[17] R Friedman, JW Morgan, On the diffeomorphism types of certain algebraic surfaces. I, J. Differential Geom. 27 (1988) 297-369 MR925124

[18] HZ Gao, Representing homology classes of almost definite 4-manifolds, Topology Appl. 52 (1993) 109-120 MR1241187

[19] R E Gompf, A new construction of symplectic manifolds, Ann. of Math. 142 (1995) 527-595 MR1356781

[20] M Gromov, Pseudoholomorphic curves in symplectic manifolds, Invent. Math. 82 (1985) 307-347 MR809718

[21] R Hind, Lagrangian isotopies in Stein manifolds arXiv:math/0311093

[22] R Hind, Lagrangian spheres in $S^{2} \times S^{2}$, Geom. Funct. Anal. 14 (2004) 303-318 MR2060197

[23] R Hind, A Ivrii, Ruled 4-manifolds and isotopies of symplectic surfaces, Math. Z. 265 (2010) 639-652 MR2644314

[24] R Hind, M Pinsonnault, W Wu, Symplectomorphism groups of non-compact manifolds and space of Lagrangians, preprint (2011) Available at http:// www. math. umn. edu/ wuxxx347

[25] H Hofer, V Lizan, J-C Sikorav, On genericity for holomorphic curves in fourdimensional almost-complex manifolds, J. Geom. Anal. 7 (1997) 149-159 MR1630789

[26] S Ivashkovich, V Shevchishin, Structure of the moduli space in a neighborhood of a cusp-curve and meromorphic hulls, Invent. Math. 136 (1999) 571-602 MR1695206

[27] K Kikuchi, Positive 2-spheres in 4-manifolds of signature $(1, n)$, Pacific J. Math. 160 (1993) 245-258 MR1233354

[28] F Lalonde, Isotopy of symplectic balls, Gromov's radius and the structure of ruled symplectic 4-manifolds, Math. Ann. 300 (1994) 273-296 MR1299063 
[29] F Lalonde, D McDuff, $J$-curves and the classification of rational and ruled symplectic 4-manifolds, from: "Contact and symplectic geometry (Cambridge, 1994)", (C B Thomas, editor), Publ. Newton Inst. 8, Cambridge Univ. Press (1996) 3-42 MR1432456

[30] E Lerman, Symplectic cuts, Math. Res. Lett. 2 (1995) 247-258 MR1338784

[31] B-H Li, T-J Li, Symplectic genus, minimal genus and diffeomorphisms, Asian J. Math. 6 (2002) 123-144 MR1902650

[32] T-J Li, Existence of symplectic surfaces, from: "Geometry and topology of manifolds", (H U Boden, I Hambleton, A J Nicas, editors), Fields Inst. Commun. 47, Amer. Math. Soc. (2005) 203-217 MR2189933

[33] T-J Li, The Kodaira dimension of symplectic 4-manifolds, from: "Floer homology, gauge theory, and low-dimensional topology", (D A Ellwood, P S Ozsváth, A I Stipsicz, Z Szabó, editors), Clay Math. Proc. 5, Amer. Math. Soc. (2006) 249-261 MR2249257

[34] T-J Li, The space of symplectic structures on closed 4-manifolds, from: "Third International Congress of Chinese Mathematicians. Part 1, 2”, (K-S Lau, Z-P Xin, S-T Yau, editors), AMS/IP Stud. Adv. Math., 42, pt. 1 2, Amer. Math. Soc. (2008) 259-277 MR2409637

[35] T-J Li, A-K Liu, The equivalence between SW and $\mathrm{Gr}$ in the case where $b^{+}=1$, Internat. Math. Res. Notices (1999) 335-345 MR1683312

[36] T-J Li, A-K Liu, Uniqueness of symplectic canonical class, surface cone and symplectic cone of 4-manifolds with $B_{+}=1$, J. Differential Geom. 58 (2001) 331-370 MR1913946

[37] T-J Li, M Usher, Symplectic forms and surfaces of negative square, J. Symplectic Geom. 4 (2006) 71-91 MR2240213

[38] T-J Li, W Wu, On homotopy types of symplectomorphism groups on close symplectic 4-manifolds, in preparation

[39] D McDuff, The structure of rational and ruled symplectic 4-manifolds, J. Amer. Math. Soc. 3 (1990) 679-712 MR1049697

[40] D McDuff, From symplectic deformation to isotopy, from: "Topics in symplectic 4manifolds (Irvine, CA, 1996)”, (R J Stern, editor), First Int. Press Lect. Ser. I, Int. Press, Cambridge, MA (1998) 85-99 MR1635697

[41] D McDuff, D Salamon, Introduction to symplectic topology, second edition, Oxford Math. Monogr., The Clarendon Press, Oxford Univ. Press, New York (1998) MR1698616

[42] D McDuff, D Salamon, J-holomorphic curves and symplectic topology, Amer. Math. Soc. Colloquium Publ. 52, Amer. Math. Soc. (2004) MR2045629

[43] D McDuff, F Schlenk, The embedding capacity of 4-dimensional symplectic ellipsoids arXiv:0912.0532 
[44] M Pinsonnault, Maximal compact tori in the Hamiltonian group of 4-dimensional symplectic manifolds, J. Mod. Dyn. 2 (2008) 431-455 MR2417479

[45] D Salamon, E Zehnder, Morse theory for periodic solutions of Hamiltonian systems and the Maslov index, Comm. Pure Appl. Math. 45 (1992) 1303-1360 MR1181727

[46] P Seidel, Symplectic automorphisms of $T^{*} S^{2}$ arXiv:math/9803084

[47] P Seidel, Lectures on four-dimensional Dehn twists, from: "Symplectic 4-manifolds and algebraic surfaces", (F Catanese, G Tian, editors), Lecture Notes in Math. 1938, Springer, Berlin (2008) 231-267 MR2441414

[48] P Seidel, I Smith, The symplectic topology of Ramanujam's surface, Comment. Math. Helv. 80 (2005) 859-881 MR2182703

[49] V Shevchishin, Secondary Stiefel-Whitney class and diffeomorphisms of rational and ruled symplectic 4-manifolds arXiv:0904.0283v2

[50] J-C Sikorav, The gluing construction for normally generic J-holomorphic curves, from: "Symplectic and contact topology: interactions and perspectives (Toronto, ON/Montreal, QC, 2001)", (Y Eliashberg, B Khesin, F Lalonde, editors), Fields Inst. Commun. 35, Amer. Math. Soc. (2003) 175-199 MR1969276

[51] C H Taubes, Counting pseudo-holomorphic submanifolds in dimension 4, J. Differential Geom. 44 (1996) 818-893 MR1438194

[52] C H Taubes, GR = SW : counting curves and connections, J. Differential Geom. 52 (1999) 453-609 MR1761081

[53] S Vidussi, Lagrangian surfaces in a fixed homology class: existence of knotted Lagrangian tori, J. Differential Geom. 74 (2006) 507-522 MR2269786

[54] J-Y Welschinger, Effective classes and Lagrangian tori in symplectic four-manifolds, J. Symplectic Geom. 5 (2007) 9-18 MR2371182

[55] C Wendl, Automatic transversality and orbifolds of punctured holomorphic curves in dimension four, Comment. Math. Helv. 85 (2010) 347-407 MR2595183

School of Mathematics, University of Minnesota

206 Church Street, Minneapolis MN 55455, USA

tjli@umn.edu, weiwei@umn.edu

www. math.umn.edu/ tjli, www.math.umn.edu/ wuxxx347

Proposed: Ronald Fintushel

Seconded: Ronald J Stern, Leonid Polterovich
Received: 30 September 2011

Revised: 15 February 2012 